\title{
Composition and quantities of dust produced by AGB-stars and returned to the interstellar medium ${ }^{\star}$
}

\author{
A. S. Ferrarotti and H.-P. Gail
}

Zentrum für Astronomie, Universität Heidelberg, Institut für Theoretische Astrophysik, Albert-Überle Str. 2, 69120 Heidelberg, Germany

e-mail: gail@ita.uni-heidelberg.de

Received 30 April 2004 / Accepted 13 September 2005

ABSTRACT

The composition of the dust mixture and the amounts of dust returned into the interstellar medium during the evolution of low and intermediate mass stars on the AGB is calculated by simple models for dust-forming stellar winds with M-, S-, and C-star chemistry. These models consider the most abundant dust species formed in circumstellar dust shells, i.e. olivine- and pyroxene-type silicate grains and iron grains in the case of M-stars, iron grains in the case of S-stars, and carbon, $\mathrm{SiC}$, and iron grains in the case of C-stars. The wind models are combined with calculations of synthetic AGB-evolution to consistently determine the variation of the dust injection rate into the interstellar medium from AGB-stars with initial mass and metallicity as the stars evolve along the AGB and change their chemical surface compositions due to repeated "dredge-up" episodes. Numerical results are presented for initial stellar masses from 1 to $7 M_{\odot}$ and for metallicities between $Z=0.001$ and $Z=0.04$, which can then be used to calculate chemical evolution models for the dust content of galaxies.

Key words. circumstellar matter - dust, extinction - stars: mass-loss - stars: winds, outflows - stars: AGB and post-AGB - evolution

\section{Introduction}

Mass-loss and dust formation accompany the late evolutionary phases of most stellar types. Part of the heavy element inventory of a star, originating from its initial equipment with such elements from the interstellar matter and from the nucleosynthesis within the star, are returned into the interstellar medium (ISM) as tiny solid particulates with the chemical composition depending on the particular elemental composition of the ejected matter in which they are formed (e.g. Gehrz 1989; Alexander 1997; Jones et al. 1997; Tielens 1999). This circumstellar dust is an essential ingredient for the galactic matter cycle since the grains serve as condensation centres for all heavy refractory elements in the ISM not yet incorporated into dust (e.g. Tielens 1998; Dwek 1998). In this way the injection of circumstellar dust into the ISM is responsible for the existence of interstellar dust.

From the various stellar types that inject dusty matter into the interstellar medium, the Asymptotic Giant Branch (AGB) stars are by far the most efficient sources of circumstellar dust. This has been found by comparing observationally based data on mass return and dust content of stellar ejecta for the different stellar sources of interstellar dust (e.g. Gehrz 1989; Alexander 1997; Tielens 1999). Other possible sources of dust for the

* Appendices A and B are only available in electronic form at http://www . edpsciences.org interstellar medium seem to be considerably less productive. This holds particularly for supernovae though they are the main contributors to the gas return into the interstellar matter and the main sources of freshly produced heavy elements.

The AGB stars and their dust injection rate into the interstellar medium therefore play a key role for the dust formation and evolution of the dust component in the galaxy. Determining the amount of dust injected by AGB stars into the ISM and its composition is a prerequisite for any attempt to model the evolution of interstellar matter in the galaxy. In this paper we therefore study the formation of dust in the circumstellar environment of AGB stars from a theoretical point of view with the aim of calculating the input data required for later model calculations of the chemical evolution of galaxies, including the dust component of their ISM by an approach like that of Dwek (1998), for instance.

The dust production rate is determined by combining simple models of dust formation in stellar winds with a synthetic AGB evolutionary model for the stars. This allows us to consistently determine the composition of the dust mixture and the quantities of dust produced by the AGB stars.

The formation of dust in the outflow from AGB stars is calculated in some detail in order to determine the composition of the mineral mixture at each instant as the star evolves along the AGB and changes its surface $\mathrm{C} / \mathrm{O}$ abundance ratio by repeated third "dredge-up" events. Equations for the formation of 
a multicomponent dust mixture of the dominating dust components in M-, S-, or C-stars are solved simultaneously with a simplified model for the stellar wind and the radiative transfer in the dust shell. This allows the dust output of the stars to be determinated for the three chemically distinct phases of AGB evolution where stars appear spectroscopically as M-, S-, or C-stars.

The evolution of the central star is followed by calculating synthetic AGB models. For the present calculation we use a rather schematic approach closely following Groenewegen \& de Jong (1993) and Marigo et al. (1996), though much improvement has been achieved in the meantime for synthetic AGB evolutionary calculations. Time-consuming calculation of many wind models required in the present model calculation to follow the luminosity variations of the star in a somewhat more simplified way.

The mass-loss rate prescription used in our synthetic AGB models is tailored for the purpose of this paper in order to properly discriminate between Miras and non-Miras with their very different stellar wind properties, which have a strong impact on the dust formation process. We take into account that Miras are found only in a limited region of the Hertzsprung-Russel diagram (Gautschy 1999).

Our approach to determining the mass-loss during the AGB evolution is similar to the approach of Schröder et al. (1999) and Schröder \& Sedlmayr (2001) but completely different with respect to dust formation. They coupled parametrized results of wind models for carbon-rich Miras (Arndt et al. 1997; Wachter et al. 2002) with a stellar evolutionary code to determine the mass-loss history and dust production of AGB stars for the range of initial masses between 1 and $2.5 M_{\odot}$. This calculation, however, does not discriminate between the different chemistries, as the $\mathrm{C} / \mathrm{O}$ abundance ratio evolves during $\mathrm{AGB}$ evolution and does not provide information on the composition of the dust mixture returned into the interstellar medium; but it is based on a more realistic treatment of AGB evolution than ours.

The plan of this paper is as follows. Section 2 describes the technical details of our model calculations of a stellar wind and of dust formation (some exemplary results for the variation of dust formation with varying $\mathrm{C} / \mathrm{O}$ abundance ratios and varying mass-loss rates are presented in Appendix A). Section 3 describes the technical details of our synthetic AGB evolutionary models and Sect. 4 describes the particular mass-loss prescriptions used for our calculations in detail. Section 5 describes how the models are calculated and how the dust production rates are determined. Section 6 presents our results for the dust production of AGB stars, and Sect. 7 gives some concluding remarks.

\section{Basic equations of dust formation}

\subsection{The model}

Condensation of dust in the circumstellar environment of AGB-star is due to the massive stellar winds that stars develop as cool giants. The winds of stars in the upper part of the AGB are thought to be driven by stellar pulsations, as the stars cross the Mira instability region in the HR-diagram. For RGB-stars of low and intermediate mass and for stars in the lower part of the AGB, the wind driving mechanism is not clear, but at this stage the stars do not form dust, or at most, form only tiny quantities of it, that are negligible for determining the total dust production of AGB stars. This evolutionary phase needs not to be considered here.

At the top of the AGB, the mass-loss rates are very high and the radiation pressure on the dust becomes the dominating driving source of the wind. The underlying stars all seem to be variables, either Miras or LPVs. The structure of the outflow is complicated in this case, as there are always a number of shocks running outwards, which are superposed on the average outflow of stellar material. This is demonstrated by several model calculations of the dust-forming stellar outflows of pulsating stars (e.g. Bowen 1988; Fleischer et al. 1991, 1992; Feuchtinger et al. 1993; Höfner \& Dorfi 1997; Höfner et al. 1998; Winters et al. 1997, 2000; Jeong et al. 2003).

Calculating dust-formation rates for multi-component dust mixtures of stars with very different elemental compositions and widely varying stellar parameters presently seems to be impossible if the shock structure of the wind is to be correctly taken into account in the model calculation. For the purpose of calculating the composition of the dust mixture and the amount of dust formed in the outflows of AGB-stars, we use a crude model that assumes stationary outflow. If one compares the velocity and density profiles of such stationary winds with published models of dust-forming pulsators (e.g. Winters et al. 2000; Jeong et al. 2003), one observes a strong resemblance in most cases to the average velocity and density profiles on which the outwards propagating shocks are superposed. We can therefore hope to at least estimate the quantities of dust formed in the outflow if dust formation is calculated for such an average outflow structure. One important consequence of this neglect of the detailed flow structure is that we cannot determine the mass-loss rate $\dot{M}$ self-consistently. We have to treat this instead as a free parameter that has to be fixed in some other way (cf. Sect. 4).

\subsubsection{Velocity and density structure}

The wind velocity is calculated from the equation of momentum conservation in the stationary case and for a spherically symmetric outflow

$v \frac{\mathrm{d} v}{\mathrm{~d} r}=-\frac{G M_{*}}{r^{2}}(1-\Gamma)$,

where pressure forces are neglected. Since we neglect the shock structure of the outflow, it would make no sense to retain the pressure forces in this equation, though this should be no problem. $M_{*}$ is the mass of the underlying star. Though considerable amounts of mass may be contained in the whole dust shell after extended periods of massive mass-loss, the dust formation is restricted to a rather narrow zone that does not contain much mass.

The quantity

$\Gamma=\frac{L_{*}}{4 \pi c G M_{*}} \kappa_{\mathrm{H}}$ 
is the ratio of radiation pressure on dust to gravitational pull of the star, where $\kappa_{\mathrm{H}}$ is the flux averaged mass extinction coefficient of the gas-dust mixture, which is dominated by the dust formed in the outflow. And $L_{*}$ is the stellar luminosity.

The equations for dust formation (see below) are solved from that point on, where the first of the dust species that dominate the opacity of the matter becomes stable. For dust-driven winds, the initial velocity at this point is chosen to be equal to the sonic velocity at this point, since for dust-driven winds the flow velocity at the inner edge of the dust shell should be about equal to the sound velocity (cf. Gail \& Sedlmayr 1985). For computational purposes a fixed value of $2 \mathrm{~km} \mathrm{~s}^{-1}$ is even more simply chosen since for dust-driven winds the properties of the model are nearly independent of the precise value of the initial velocity. This initial velocity is held fixed up to the point where $\Gamma$ exceeds unity, and from this point on Eq. (1) is solved for $v$. Since nearly all of the dust is formed in the region where $\Gamma>1$, the solution for the flow structure only weakly depends on how $v$ is determined in the region $\Gamma<1$. This simplified procedure was already used in our preceding papers (Gail \& Sedlmayr 1999; Ferrarotti \& Gail 2001, 2002, 2003), and it allows a simple and efficient calculation of rather complex dustformation models.

The density structure in the wind is calculated from the equation of mass conservation

$\dot{M}=4 \pi r^{2} \rho v$.

$\dot{M}$ is the mass-loss rate, which is considered to be a free parameter of the problem in our calculation of dust formation. In our later calculations of synthetic AGB models, the mass-loss rate is specified as described in Sect. 4.

\subsubsection{Temperature structure}

The temperature structure in the wind is calculated in the approximation of Lucy $(1971,1976)$ for a spherically symmetric grey circumstellar dust shell from

$T^{4}=\frac{1}{2} T_{\mathrm{eff}}^{4}\left[1+\sqrt{1-\frac{R_{*}^{2}}{r^{2}}}+\frac{3}{2} \tau_{\mathrm{L}}\right]$,

where $\tau_{\mathrm{L}}$ is defined by

$\frac{\mathrm{d} \tau_{\mathrm{L}}}{\mathrm{d} r}=-\rho \kappa_{\mathrm{H}} \frac{R_{*}^{2}}{r^{2}}$

and

$\lim _{r \rightarrow \infty} \tau_{\mathrm{L}}=0$,

where $T_{\text {eff }}$ is the effective temperature of the stellar atmosphere, $R_{*}$ the (fictitious) stellar radius determined from the relation

$4 \pi R_{*}^{2} \sigma_{\mathrm{SB}} T_{\mathrm{eff}}^{4}=L_{*}$,

and $\sigma_{\mathrm{SB}}$ is the Stefan-Boltzmann constant.

The whole wind model is a simplified version of our former stationary wind models for the dust shells of carbon-rich AGB stars (Gail \& Sedlmayr 1987, 1988; Dominik 1990).

\subsubsection{Stellar parameters}

The stellar parameters $M_{*}, L_{*}$, and $T_{\text {eff }}$ are given later by the calculation of synthetic AGB evolutionary models.

\subsubsection{Dust opacity and the Eddington limit}

For dust-forming stars the radiation pressure on grains exceeds the gravitational attraction of the star, if the stellar luminosity exceeds the Eddington limit luminosity

$L_{\mathrm{Edd}}=\frac{4 \pi c G M_{*}}{\kappa_{\mathrm{H}}}$.

If $L>L_{\text {Edd }}$, a stellar wind may be driven by dust condensation. If dust is present, the opacity is dominated by the few abundant dust species formed in the outflow. In our model calculation, we approximate the flux-averaged mass extinction coefficient $\kappa_{\mathrm{H}}$ in Eq. (2) by

$\kappa_{\mathrm{H}}=\kappa_{\mathrm{gas}}+\sum_{i} f_{i} \kappa_{i, \mathrm{dust}}$

The summation is over all abundant dust species $i$ that contribute significantly to the total opacity. In our model calculations, we consider silicates and iron in the case of M-stars, iron in the case of S-stars, and carbon, $\mathrm{SiC}$, and iron in the case of $\mathrm{C}$-stars for calculating the radiative acceleration of the wind material.

Equation (9) assumes that the contributions of all the species simply add up, which generally is not valid for the fluxaveraged mean extinction, except in the case of grey extinction. Since the dust extinction varies only slowly with wavelength in the wavelength region where most of the stellar energy is emitted (around $\approx 1.0 \ldots 1.5 \mu \mathrm{m}$ ), the dust absorbs nearly grey, and usually one dust species dominates the extinction. The calculation of $\kappa$ by simply adding up the separate contributions of the different dust species is then sufficiently accurate.

The contributions $\kappa_{i \text {,dust }}$ for all dust species $i$ are approximated by their Rosseland mean opacities. The $\kappa_{i \text {,dust }}$ are determined for complete condensation of the key element ${ }^{1}$ required to form the particular dust species $i$ into this kind of dust. The degree of condensation $f_{i}$ of the key element into dust species $i$ in Eq. (9) then accounts for the true abundance of dust species $i$. The various approximations used for calculating $\kappa_{i, \text { dust }}$ are described in Gail \& Sedlmayr (1999) and Ferrarotti $\&$ Gail (2001, 2002). For the silicates we preliminarily use the opacity for circumstellar silicate dust derived by Ossenkopf et al. (1992) in the present calculation, since this seems to yield more reliable wind models of oxygen-rich stars (cf. Joeng et al. 2003) than does using opacities of pure silicates, which tend to have opacities that are much too low in the mid-infrared region.

Figure 1 shows the limit luminosity $L_{\text {Edd }}$ corresponding to the extinction of a single dust species for the dust materials considered in this paper. There $L_{\text {Edd }}$ is calculated for a stellar mass of one $M_{\odot}$ and a degree of condensation of $f=0.5$. For

\footnotetext{
${ }^{1}$ We denote as key element the least abundant of the elements required to form the dust species under consideration. The amounts of dust that can be formed for this dust species are limited by the abundance of the key element.
} 


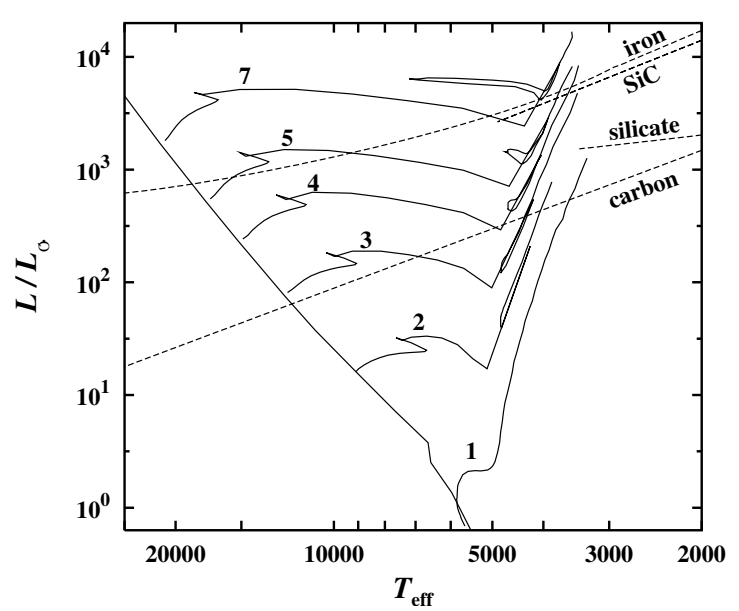

Fig. 1. Eddington limit luminosity $L_{\text {Edd }}$ for the main dust components (dashed lines). The limit luminosity is calculated for a stellar mass of $M_{*}=1 M_{\odot}$ and a degree of condensation of the key element of $f=0.5$. For other values of $M_{*}$ and $f$ the limit luminosity scales as $M_{*} / f$. Full lines show evolutionary tracks of Schaller et al. (1992) for the indicated initial stellar masses. The upper end of the evolutionary tracks corresponds to the onset of thermal pulsing on the AGB of Pop I stars (tip of RGB for $M_{*}=1,2 M_{\odot}$ ).

comparison the figure also shows some evolutionary tracks of stars with initial masses of 2 to $7 M_{\odot}$ up to the point where thermal pulsing on the AGB starts to operate. The figure shows that the formation of dust becomes dynamically important for a stellar outflow, if the stellar luminosity of giant stars exceeds a few $10^{3} L_{\odot}$, since from that point on $L>L_{\text {Edd }}$.

\subsection{Dust formation in M-stars}

The dust mixture expected to be formed in an oxygen-rich element mixture and the basic set of equations to be solved for condensation of dust in this case was discussed in Gail $\&$ Sedlmayr (1998a, 1999). The case of the condensation of pyroxene-type dust that was not considered in that paper was treated in Ferrarotti \& Gail (2001). In the present calculation we restrict the calculation to the four dust species olivine-type ${ }^{2}$ dust, pyroxene-type dust, quartz-type dust, and metallic iron. Other dust species considered in our previous calculations are found to be of low abundance. In the following we briefly list the relevant equations used for calculating the growth of the different species. For details see Gail \& Sedlmayr (1999) and Ferrarotti \& Gail (2001, 2002).

\subsubsection{Formation of olivine-type dust}

The growth of olivine-type dust grains with the composition $\mathrm{Mg}_{2 x} \mathrm{Fe}_{2(1-x)} \mathrm{SiO}_{4}$ is assumed to proceed via the following

\footnotetext{
${ }^{2}$ We follow a suggestion by A. P. Jones (2000) to discriminate between dust species' with chemical composition such as that of olivine, but with an unknownor amorphous lattice structure, from the true crystalline mineral olivine by calling them olivine-type dust (and correspondingly in other cases) and use mineral names only if it is known that the dust species is a genuine mineral.
}

overall reaction

$$
\begin{aligned}
2 x \mathrm{Mg}+2(1-x) \mathrm{Fe}+\mathrm{SiO}+ & 3 \mathrm{H}_{2} \mathrm{O} \longrightarrow \\
& \mathrm{Mg}_{2 x} \mathrm{Fe}_{2(1-x)} \mathrm{SiO}_{4}(s)+3 \mathrm{H}_{2} .
\end{aligned}
$$

The rate-determining step of the reaction is assumed to be i) either the addition of $\mathrm{SiO}$ molecules; ii) the addition of $\mathrm{Mg}+\mathrm{Fe}$ atoms from the gas phase to the grain, depending on whether sufficient $\mathrm{Mg}+\mathrm{Fe}$ is available to provide two $\mathrm{Mg}+\mathrm{Fe}$ atoms for each $\mathrm{SiO}$ or not; or iii) the supply of oxygen by $\mathrm{H}_{2} \mathrm{O}$ molecules in order to oxidize $\mathrm{SiO}$ to the $\mathrm{SiO}_{4}$-tetrahedron in olivine-type dust. Especially if the $\mathrm{C} / \mathrm{O}$ abundance ratio approaches unity, the formation of olivine-type dust is limited by the paucity of available $\mathrm{O}$. The change of particle radius $a$ by growth or decomposition is given by

$$
\frac{\mathrm{d} a_{\mathrm{ol}}}{\mathrm{d} t}=V_{0, \mathrm{ol}}\left(J_{\mathrm{ol}}^{\mathrm{gr}}-J_{\mathrm{ol}}^{\mathrm{dec}}\right)
$$

where the total growth rate of the grains is

$$
J_{\mathrm{ol}}^{\mathrm{gr}}=\min \left(J_{\mathrm{SiO}}^{\mathrm{gr}}, \frac{1}{2}\left[J_{\mathrm{Mg}}^{\mathrm{gr}}+J_{\mathrm{Fe}}^{\mathrm{gr}}\right], \frac{1}{3} J_{\mathrm{H}_{2} \mathrm{O}}^{\mathrm{gr}}\right),
$$

where $V_{0, o l}$ is the volume of the nominal molecule in the solid. The equation for the magnesium content $x$ of the olivine-type grains is

$$
\frac{\mathrm{d} x_{\mathrm{ol}}}{\mathrm{d} t}=\frac{3 V_{0, \mathrm{ol}}}{a_{\mathrm{ol}}}\left[\left(x_{\mathrm{g}}-x_{\mathrm{ol}}\right) J_{\mathrm{ol}}^{\mathrm{gr}}+\frac{1}{2}\left(J_{+}^{\mathrm{ex}}-J_{-}^{\mathrm{ex}}\right)\right] .
$$

For the definitions of the growth rates $J_{\mathrm{SiO}}^{\mathrm{gr}}, J_{\mathrm{Mg}}^{\mathrm{gr}}, J_{\mathrm{Fe}}^{\mathrm{gr}}, J_{\mathrm{H}_{2} \mathrm{O}}^{\mathrm{gr}}$, the decomposition rate $J_{\mathrm{ol}}^{\mathrm{dec}}$, and the exchange rates $J_{+}^{\mathrm{ex}}, J_{-}^{\mathrm{ex}}$ for olivine-type grains, see Gail \& Sedlmayr (1999). There $x_{g}$ is defined as

$x_{\mathrm{g}}=\frac{\epsilon_{\mathrm{Mg}, \text { gas }}}{\epsilon_{\mathrm{Mg}, \text { gas }}+\epsilon_{\mathrm{Fe}, \text { gas }}}$,

where $\epsilon_{\mathrm{Mg} \text {, gas }}$ and $\epsilon_{\mathrm{Fe} \text {, gas }}$ are the actual abundances of $\mathrm{Mg}$ and $\mathrm{Fe}$ in the gas phase.

To save computing time, we do not consider calculation of the diffusion of $\mathrm{Mg}^{2+}$ and $\mathrm{Fe}^{2+}$ cations in the present model, though this might be important as shown in Gail \& Sedlmayr (1999). The grains are assumed to have an amorphous lattice structure. Observationally it is known that crystalline silicate grains are formed in some circumstellar dust shells (see Molster et al. 2002a,b,c), but this is neglected in this paper.

Grain drift velocities become supersonical only for massloss rates that are significantly less than $10^{-5} M_{\odot} \mathrm{yr}^{-1}$ (e.g. Gail \& Sedlmayr 1985). Grain drift therefore is presently not considered since it is not important during the superwind phase, where nearly all of the dust is formed that will be formed during the AGB evolution of a star.

\subsubsection{Formation of pyroxene-type dust}

The growth of pyroxene-type dust grains of the composition $\mathrm{Mg}_{x} \mathrm{Fe}_{1-x} \mathrm{SiO}_{3}$ is assumed to proceed via the following 
overall reaction

$x \mathrm{Mg}+(1-x) \mathrm{Fe}+\mathrm{SiO}+2 \mathrm{H}_{2} \mathrm{O} \longrightarrow$

$$
\mathrm{Mg}_{x} \mathrm{Fe}_{1-x} \mathrm{SiO}_{3}(s)+2 \mathrm{H}_{2} .
$$

The rate-determining step of the reaction again is assumed to be either the addition of $\mathrm{SiO}$ molecules, the addition of $\mathrm{Mg}+\mathrm{Fe}$ atoms from the gas phase to the grain, or the supply of oxygen by $\mathrm{H}_{2} \mathrm{O}$ molecules in order to oxidize $\mathrm{SiO}$ to the $\mathrm{SiO}_{4}$-tetrahedron in pyroxene-type dust. The change of particle radius $a$ by growth or decomposition is given by

$\frac{\mathrm{d} a_{\mathrm{py}}}{\mathrm{d} t}=V_{0, \mathrm{py}}\left(J_{\mathrm{py}}^{\mathrm{gr}}-J_{\mathrm{py}}^{\mathrm{dec}}\right)$,

where the total growth rate of the grains is

$J_{\text {py }}^{\mathrm{gr}}=\min \left(J_{\mathrm{SiO}}^{\mathrm{gr}},\left[J_{\mathrm{Mg}}^{\mathrm{gr}}+J_{\mathrm{Fe}}^{\mathrm{gr}}\right], \frac{1}{2} J_{\mathrm{H}_{2} \mathrm{O}}^{\mathrm{gr}}\right)$.

The equation for the magnesium content $x$ of the pyroxene-type grains is

$\frac{\mathrm{d} x_{\mathrm{py}}}{\mathrm{d} t}=\frac{3 V_{0, \mathrm{py}}}{a_{\mathrm{py}}}\left[\left(x_{g}-x_{\mathrm{py}}\right) J_{\mathrm{py}}^{\mathrm{gr}}+\left(J_{+}^{\mathrm{ex}}-J_{-}^{\mathrm{ex}}\right)\right]$.

For the definitions of the growth rates $J_{\mathrm{SiO}}^{\mathrm{gr}}, J_{\mathrm{Mg}}^{\mathrm{gr}}, J_{\mathrm{Fe}}^{\mathrm{gr}}$, for the decomposition rate $J_{\mathrm{py}}^{\mathrm{dec}}$, and for the exchange rates $J_{+}^{\mathrm{ex}}, J_{-}^{\mathrm{ex}}$ for pyroxene-type dust, see Ferrarotti \& Gail (2001). Also, for pyroxene-type dust we assume the grains to have an amorphous lattice structure.

\subsubsection{Formation of quartz-type dust}

The growth of quartz-type dust grains with composition $\mathrm{SiO}_{2}$ is assumed to proceed via the following over-all reaction

$\mathrm{SiO}+\mathrm{H}_{2} \mathrm{O} \longrightarrow \mathrm{SiO}_{2}(s)+\mathrm{H}_{2}$.

The rate-determining step of the reaction is assumed to be the addition of the less abundant of the two growth species $\mathrm{SiO}$ and $\mathrm{H}_{2} \mathrm{O}$. The change of particle radius $a$ by growth or decomposition is given by

$\frac{\mathrm{d} a_{\mathrm{qu}}}{\mathrm{d} t}=V_{0, \mathrm{qu}}\left(J_{\mathrm{qu}}^{\mathrm{gr}}-J_{\mathrm{qu}}^{\mathrm{dec}}\right)$,

where the total growth rate of the grains is

$J_{\mathrm{qu}}^{\mathrm{gr}}=\min \left(J_{\mathrm{SiO}}^{\mathrm{gr}}, J_{\mathrm{H}_{2} \mathrm{O}}^{\mathrm{gr}}\right)$.

For the definitions of the growth rates $J_{\mathrm{SiO}}^{\mathrm{gr}}, J_{\mathrm{H}_{2} \mathrm{O}}^{\mathrm{gr}}$ and the decomposition rate $J_{\text {qu }}^{\text {dec }}$, see Gail \& Sedlmayr (1999) and Ferrarotti \& Gail (2002).

\subsubsection{Formation of iron dust}

The change of particle radius $a$ of iron grains by growth or vaporization is given by

$\frac{\mathrm{d} a_{\text {ir }}}{\mathrm{d} t}=V_{0, \text { ir }}\left(J_{\text {ir }}^{\mathrm{gr}}-J_{\text {ir }}^{\mathrm{vap}}\right)$.
For the definitions of the growth rate $J_{\text {ir }}^{\mathrm{gr}}$ and the vaporization rate $J_{\text {ir }}^{\text {vap }}$, see Gail \& Sedlmayr (1999) and Ferrarotti \& Gail (2002).

\subsubsection{Initial conditions}

It is assumed that the dust grains grow on some kind of seed nuclei that are formed prior to the point where the different dust components start to condense. These could be, for instance, small $\mathrm{TiO}_{2}$ grains, as proposed in Gail \& Sedlmayr (1998b). This is analogous to the case of carbon grains, where laboratory investigations of presolar grains have shown that many of them grew around seed nuclei of TiC or solid solutions of $\mathrm{TiC}$ with $\mathrm{ZrC}$ and MoC (cf. Bernatowicz \& Cowsik 1997). Semiempirical calculations of the nucleation rate of $\mathrm{TiO}_{2}$ seed particles by Gail \& Sedlmayr (1998b) and recent model calculations of stellar outflows by Jeong et al. (2003) based on more detailed quantum mechanical calculations of the nucleation rate of $\mathrm{TiO}_{2}$ have shown that such grains may indeed be the required seed nuclei.

The radii of these seed nuclei (denoted by $a_{0, \mathrm{ol}} \ldots$ ) and their particle densities (denoted by $n_{d, \mathrm{ol}} \ldots$ ) can in principle be calculated from the theory of dust nucleation for $\mathrm{TiO}_{2}$ clusters. Since, however, $\mathrm{TiO}_{2}$ clusters have not yet been definitely confirmed as seed nuclei, we introduce some assumptions on these quantities. As the final results for the grain radii are nearly independent of the particular assumption on $a_{0}$ (as long as reasonable values are chosen), we arbitrarily assume $a_{0}=1 \mathrm{~nm}$ for the radii of the seed nuclei.

The equations for the grain radii $a_{\mathrm{ol}}, a_{\mathrm{py}}, a_{\mathrm{qu}}, a_{\mathrm{ir}}$ are solved from that point on with the initial condition $a=a_{0}$, where the activity of this compound in the cooling flow first exceeds unity (cf. Gail 2003). For an oxygen-rich mixture, this condition is met first by olivine-type dust, then by pyroxene-type dust, quartz-type dust, and finally by solid iron grains.

Simultaneously with the equations for the particle radii, the equations for the magnesium contents $x_{\mathrm{ol}}, x_{\mathrm{py}}$ of olivine- and pyroxene-type dust are solved with the fixed initial conditions $x=0.8$. The concept for determining initial conditions described in Gail \& Sedlmayr (1999) is not used in the present calculation since, again, the final results are nearly independent of the assumption on the initial condition, provided the initial value of $x$ is not too small. It should be noted that the temperature for the onset of silicate condensation depends considerably on $x$ (cf. Fig. 1 in Gail \& Sedlmayr 1999), so that the initial value of $x$ must not be chosen so small that the stability limit of silicate dust approaches the stability limit of solid iron, upon which the results would become unrealistic. The above choice for the initial value yields results that are not distinguishable from results obtained if the initial condition is determined in a self-consistent way.

\subsubsection{Degree of condensation}

The degrees of condensation of the key elements for the different species are required for calculating the opacity and the depletion of the gas phase from condensible elements. 
For the silicate compounds, we take $\mathrm{Si}$ as the key element in describing how much dust is formed. For olivine-type dust, the fraction of the $\mathrm{Si}$ condensed into dust grains is

$f_{\mathrm{ol}}=\frac{4 \pi\left(a_{\mathrm{ol}}^{3}-a_{0, \mathrm{ol}}^{3}\right)}{3 V_{0, \mathrm{ol}}} \frac{n_{d, \mathrm{ol}}}{\epsilon_{\mathrm{Si}} N_{\mathrm{H}}}$.

The degree of condensation of $\mathrm{Si}$ in pyroxene-type dust is

$f_{\mathrm{py}}=\frac{4 \pi\left(a_{\mathrm{py}}^{3}-a_{0, \mathrm{py}}^{3}\right)}{3 V_{0, \mathrm{py}}} \frac{n_{d, \mathrm{py}}}{\epsilon_{\mathrm{Si}} N_{\mathrm{H}}}$,

and the degree of condensation of $\mathrm{Si}$ in quartz-type dust is

$f_{\mathrm{qu}}=\frac{4 \pi\left(a_{\mathrm{qu}}^{3}-a_{0, \mathrm{qu}}^{3}\right)}{3 V_{0, \mathrm{qu}}} \frac{n_{d, \mathrm{qu}}}{\epsilon_{\mathrm{Si}} N_{\mathrm{H}}}$.

For iron grains, Fe is the key element. The degree of condensation of $\mathrm{Fe}$ in iron grains is

$f_{\mathrm{ir}}=\frac{4 \pi\left(a_{\mathrm{ir}}^{3}-a_{0, \mathrm{ir}}^{3}\right)}{3 V_{0, \mathrm{ir}}} \frac{n_{d, \mathrm{ir}}}{\epsilon_{\mathrm{Fe}} N_{\mathrm{H}}}$,

where $n_{d}$ is the number density of seed nuclei of the different dust species. In the model calculations we assume $n_{d}=3 \times$ $10^{-13} \cdot N_{\mathrm{H}}$ for all dust species (see Ferrarotti \& Gail 2003), where $N_{\mathrm{H}}$ is the number of hydrogen nuclei, which is determined from the mass density $\rho$ by

$N_{\mathrm{H}}=\frac{\rho}{\left(1+4 \epsilon_{\mathrm{He}}\right) m_{\mathrm{H}}}$.

\subsubsection{Change of gas phase abundances}

For the growth species $\mathrm{SiO}, \mathrm{Mg}, \mathrm{Fe}$, and $\mathrm{H}_{2} \mathrm{O}$, we can assume that $\mathrm{Mg}$ and $\mathrm{Fe}$ are present in the gas phase only as free atoms, that $\mathrm{Si}$ is present in the gas phase only as $\mathrm{SiO}$ (we neglect that a small fraction of $\mathrm{Si}$ is present as $\mathrm{SiS}$ ), and that oxygen is present in the gas phase only as $\mathrm{CO}, \mathrm{SiO}$, and $\mathrm{H}_{2} \mathrm{O}$.

The particle density of $\mathrm{H}_{2} \mathrm{O}$ molecules in the gas phase is then given by

$n_{\mathrm{H}_{2} \mathrm{O}}=\left[\epsilon_{\mathrm{O}}-\epsilon_{\mathrm{C}}-\left(1+3 f_{\mathrm{ol}}+2 f_{\mathrm{py}}+f_{\mathrm{qu}}\right) \epsilon_{\mathrm{Si}}\right] N_{\mathrm{H}}$,

the particle density of $\mathrm{SiO}$ molecules by

$n_{\mathrm{SiO}}=\left(1-f_{\mathrm{ol}}-f_{\mathrm{py}}-f_{\mathrm{qu}}\right) \epsilon_{\mathrm{Si}} N_{\mathrm{H}}$,

the particle density of Fe atoms by

$n_{\mathrm{Fe}}=\epsilon_{\mathrm{Fe}, \text { gas }} N_{\mathrm{H}}$,

and the particle density of $\mathrm{Mg}$ atoms by

$n_{\mathrm{Mg}}=\epsilon_{\mathrm{Mg}, \mathrm{gas}} N_{\mathrm{H}}$,

where

$\epsilon_{\mathrm{Fe}, \text { gas }}=\epsilon_{\mathrm{Fe}}\left(1-f_{\text {ir }}\right)$

$$
-\left(2\left(1-x_{\mathrm{ol}}\right) f_{\mathrm{ol}}+\left(1-x_{\mathrm{py}}\right) f_{\mathrm{py}}\right) \epsilon_{\mathrm{Si}}
$$

$\epsilon_{\mathrm{Mg}, \text { gas }}=\epsilon_{\mathrm{Mg}}-\left(2 x_{\mathrm{ol}} f_{\mathrm{ol}}+x_{\mathrm{py}} f_{\mathrm{py}}\right) \epsilon_{\mathrm{Si}}$.

These particle densities are used for calculating the growth rates of the different dust species.
Table 1. Element abundances by number relative to $\mathrm{H}$ used in the model computations. The second column shows results of Reddy et al. (2003), the third column the solar abundances of Grevesse \& Sauval (1998) with the correction for O by Allende Prieto et al. (2001) and for $\mathrm{C}$ by Allende Prieto et al. (2002).

\begin{tabular}{lcc}
\hline \hline Element & $\epsilon$ & $\epsilon_{\odot}$ \\
\hline $\mathrm{He}$ & - & $9.77 \times 10^{-2}$ \\
$\mathrm{C}$ & $3.24 \times 10^{-4}$ & $2.45 \times 10^{-4}$ \\
$\mathrm{~N}$ & $1.15 \times 10^{-4}$ & $8.32 \times 10^{-5}$ \\
$\mathrm{O}$ & $5.37 \times 10^{-4}$ & $4.89 \times 10^{-4}$ \\
$\mathrm{Ne}$ & - & $1.20 \times 10^{-4}$ \\
$\mathrm{Mg}$ & $3.47 \times 10^{-5}$ & $3.80 \times 10^{-5}$ \\
$\mathrm{Si}$ & $4.17 \times 10^{-5}$ & $3.55 \times 10^{-5}$ \\
$\mathrm{~S}$ & $2.19 \times 10^{-5}$ & $2.14 \times 10^{-5}$ \\
$\mathrm{Fe}$ & $2.82 \times 10^{-5}$ & $3.18 \times 10^{-5}$ \\
\hline
\end{tabular}

\subsection{Dust formation in $C$-stars}

The case of C-stars is treated in a similar way as the case of M-stars. The dust species considered in this case are solid carbon, $\mathrm{SiC}$, and solid iron. The MgS dust observed in part of the carbon stars could not be included in this model calculation, since its formation mechanism in the shells of C-stars is not known. In any case, $\mathrm{MgS}$ dust cannot be important for driving the wind since the $\mathrm{S}$ abundance for carbon stars is lower by at least a factor of 20 than the $\mathrm{C}$ abundance once the $\mathrm{C}$ abundance significantly exceeds the $\mathrm{O}$ abundance (see Table 1 ). Also other dust species may exist, but at present we restrict our calculations to those dust species that are expected to be the most abundant ones and that presumably are present in all carbon stars.

\subsubsection{Formation of carbon dust}

The most abundant carbon-bearing species in the gas phase besides $\mathrm{CO}$ is $\mathrm{C}_{2} \mathrm{H}_{2}$. We assume that the growth of carbon proceedes via the net reaction

$\mathrm{C}_{2} \mathrm{H}_{2} \longrightarrow 2 \mathrm{C}(s)+\mathrm{H}_{2}$.

The details of the growth process are rather complicated (e.g. Goeres 1993; Cherchneff 1998) and are not discussed here. A growth of carbon grains is not possible just below the stability limit of solid carbon in the element mixture of $\mathrm{C}$ stars, which would be at about $1600 \mathrm{~K}$, but only at a considerably lower temperature, below $1100 \mathrm{~K}$, because one of the key steps of $\mathrm{C}_{2} \mathrm{H}_{2}$ addition does not work at higher temperatures (Cherchneff et al. 1992). Condensation temperatures of this order are usually also found from the IR emission from circumstellar dust shells of carbon stars (e.g. Kerschbaum 1999; Groenewegen 1995; Groenewegen et al. 1998; Bagnulo et al. 1998), though the scattering of data is considerable and the accuracy of observationally derived temperatures of dust at the inner boundary of dust shells around carbon stars cannot be considered as high. We simply approximate carbon growth by the assumption that growth starts at $1100 \mathrm{~K}$ and that there is no 
corresponding destruction reaction. The equation of growth for carbon dust grains is

$\frac{\mathrm{d} a_{\mathrm{ca}}}{\mathrm{d} t}=V_{0, \mathrm{ca}} J_{\mathrm{ca}}^{\mathrm{gr}}$.

The growth rate $J_{\text {ca }}^{\text {gr }}$ is defined as analogous to that of other dust species (cf. Gail \& Sedlmayr 1999; Ferrarotti \& Gail 2001, 2002).

Laboratory investigations of presolar carbon grains from circumstellar dust shells of $\mathrm{C}$ stars show that many of them grow around $n \mathrm{~m}$-sized seed particles of $\mathrm{TiC}$ or solid solutions of $\mathrm{TiC}$ with $\mathrm{ZrC}$ and $\mathrm{MoC}$. Since carbon grows under strong non-equilibrium conditions, its growth starts at temperatures well below the condensation temperature of $\mathrm{TiC}$, which is discussed in Bernatowicz et al. (1996) and Lodders \& Fegley $(1995)^{3}$. Most of the grains grow, however, around $n$ m-sized disordered stacks of what seems to be clusters of PAHs. In our model calculations we simply assume, that such seed particles already exist if massive carbon dust condensation becomes possible.

\subsubsection{Formation of SiC-type dust}

The growth of $\mathrm{SiC}$ is assumed to proceed via the overall reaction

$2 \mathrm{Si}+\mathrm{C}_{2} \mathrm{H}_{2} \longrightarrow 2 \mathrm{SiC}(s)+\mathrm{H}_{2}$.

The rate-determining step of the reaction is assumed to be the addition of the less abundant of the two growth species $\mathrm{Si}$ and $\mathrm{C}_{2} \mathrm{H}_{2}$. The change of particle radius $a$ by growth or decomposition is given by

$\frac{\mathrm{d} a_{\mathrm{sc}}}{\mathrm{d} t}=V_{0, \mathrm{sc}}\left(J_{\mathrm{sc}}^{\mathrm{gr}}-J_{\mathrm{sc}}^{\mathrm{dec}}\right)$,

where the total growth rate of the grains is

$J_{\mathrm{sc}}^{\mathrm{gr}}=\min \left(J_{\mathrm{Si}}^{\mathrm{gr}}, J_{\mathrm{C}_{2} \mathrm{H}_{2}}^{\mathrm{gr}}\right)$.

For definitions of the growth rates $J_{\mathrm{SiO}}^{\mathrm{sc}}, J_{\mathrm{C}_{2} \mathrm{H}_{2}}^{\mathrm{gr}}$ and the decomposition rate $J_{\mathrm{sc}}^{\mathrm{dec}}$, see Ferrarotti \& Gail (2002).

\subsubsection{Supplementary equations}

The equation for the degree of condensation of $\mathrm{C}$ in carbon grains is

$f_{\mathrm{ca}}=\frac{4 \pi\left(a_{\mathrm{ca}}^{3}-a_{0, \mathrm{ca}}^{3}\right)}{3 V_{0, \mathrm{ca}}} \frac{n_{d, \mathrm{ca}}}{\epsilon_{\mathrm{C}} N_{\mathrm{H}}}$,

and the degree of condensation of $\mathrm{Si}$ in $\mathrm{SiC}$ grains is

$f_{\mathrm{sc}}=\frac{4 \pi\left(a_{\mathrm{sc}}^{3}-a_{0, \mathrm{sc}}^{3}\right)}{3 V_{0, \mathrm{sc}}} \frac{n_{d, \mathrm{sc}}}{\epsilon_{\mathrm{Si}} N_{\mathrm{H}}}$.

The carbon not bound in $\mathrm{CO}$ or in solid carbon and $\mathrm{SiC}$ is present in the gas phase as $\mathrm{C}_{2} \mathrm{H}_{2}$. Other C-bearing gas

3 Note, however, that carbon does not condense in chemical equilibrium, as is assumed in that paper. This assumption is at odds with observed condensation temperatures. phase species can essentially be neglected. The particle density of $\mathrm{C}_{2} \mathrm{H}_{2}$ then is

$n_{\mathrm{C}_{2} \mathrm{H}_{2}}=\left(\left(1-f_{\mathrm{ca}}\right) \epsilon_{\mathrm{C}}-\epsilon_{\mathrm{O}}-\left(1-f_{\mathrm{sc}}\right) \epsilon_{\mathrm{Si}}\right) N_{\mathrm{H}}$.

In a carbon-rich element mixture, the silicon in the gas phase is bound in $\mathrm{SiS}$, and any excess of $\mathrm{Si}$ is present as the free atom. Other Si-bearing species like $\mathrm{SiC}_{2}$ have smaller abundances and are neglected in this calculation. Then we have, for the abundance of free $\mathrm{Si}$ atoms,

$n_{\mathrm{Si}}=\left(\left(1-f_{\mathrm{sc}}\right) \epsilon_{\mathrm{Si}}-\epsilon_{\mathrm{S}}\right) N_{\mathrm{H}}$.

These particle densities are then used for calculating the dust growth rates.

\subsection{Dust formation in S-stars}

The dust mixture expected to be formed in an element mixture where the $\mathrm{C} / \mathrm{O}$ abundance ratio is close to unity, and the basic set of equations to be solved for dust condensation in this case was discussed in Ferrarotti \& Gail (2002). The precise definition for which stars to consider as $\mathrm{S}$ star in our calculation is given in Sect. A.1.

For simplicity we only consider the condensation of metallic iron and quartz-type dust. The species FeSi is not considered separately but is lumped together with the iron. The condensation of solid iron is essentially independent of the $\mathrm{C} / \mathrm{O}$ abundance ratio. Its formation and the formation of quartz are treated as in the case of M-stars.

In Ferrarotti \& Gail (2002) silicon carbide was also found to be a likely condensate for part of the $\mathrm{S}$ stars, but only if the carbon abundance exceeds the critical value

$\epsilon_{\mathrm{C}}=\epsilon_{\mathrm{O}}-\epsilon_{\mathrm{Si}}+\epsilon_{\mathrm{S}}$,

because only then is some carbon left over in the gas phase, which is not bound in $\mathrm{CO}$ and $\mathrm{SiO}$. This limit is very close to a $\mathrm{C} / \mathrm{O}$ abundance ratio of unity, i.e. such stars are at the very verge of the $\mathrm{C} / \mathrm{O}$ abundance ratios where they spectrally appear as type $\mathrm{S}$ or already as the transition type SC. We neglect the possible formation of $\mathrm{SiC}$ type dust in $\mathrm{S}$ stars because the $\mathrm{SiC}$ dust production of that small fraction of $\mathrm{S}$ stars can be neglected as compared to $\mathrm{SiC}$ dust production by $\mathrm{C}$ stars.

\subsection{Method of solution}

The coupled system of differential equations for the wind and dust formation is solved with an Adams-Bashforth integrator (e.g. Golub \& Ortega 1992), which requires only one evaluation of the right hand sides of the differential equations at each time step but is of third-order accuracy. The equations are solved in the radially outward direction. We do not determine the onset of dust formation by a separate procedure, as in our previous calculations, but start the integrations at a somewhat smaller radius than the one where the first dust species starts to grow. The r.h.s of the equations of dust formation initially is set to zero, as long as the rates of dust vaporization $J^{\text {vap }}$ or decomposition $J^{\text {dec }}$ exceed the corresponding growth rates $J^{\mathrm{gr}}$. Dust formation is then automatically switched on during the 
course of integrations. The outer radius in all cases is chosen as $10^{4} R_{*}$.

Equation (5) for $\tau_{\mathrm{L}}$ has its boundary condition prescribed at the outer boundary of the integration interval. This boundary condition (6) is satisfied by a multiple shooting method; i.e. we estimate an initial value of $\tau_{\mathrm{L}}$ at the inner boundary of the dust shell and vary this initial value until the final value of the solution of Eq. (5) satisfies Eq. (6) at the outer boundary of the dust shell.

The time required for calculating a model for a circumstellar dust shell is about $1 \mathrm{~s}$ on a PC running with $2 \mathrm{GHz}$. This is sufficiently fast that a number of dust-formation models can be calculated at each timestep in the synthetic AGB evolution model; i.e. no interpolation from tabulated solutions of wind models or analytic fits to a set of solutions are necessary.

\subsection{Models with constant outflow velocity}

For low mass-loss rates only small amounts of dust are formed and $\Gamma$, as defined by Eq. (2), remains $\ll 1$. Radiation pressure on dust is dynamically unimportant in this case, and the stellar wind is driven by some other mechanism, for instance by pulsations alone. In such cases the main acceleration mechanism for the outflow operates close to the star and not within the dust condensation zone. The wind then already flows with supersonic velocities into the condensation zone (for typical velocity profiles see Harper 1996). The best approximation for calculating dust-formation models in such cases is to hold the velocity fixed across the condensation zone. Our subroutine package allows us to treat such cases by replacing the rhs of Eq. (1) by zero, which then simply conserves the initial value of the velocity.

\section{Synthetic AGB-evolution}

In order to determine the amount of gas and dust returned by low and medium mass stars during their evolution on the AGB, we use synthetic models of AGB-evolution. Such models have been used by various authors to study the amounts of freshly synthesized nuclei returned into the interstellar medium or for population synthesis purposes (e.g. Renzini \& Voli 1981; Groenewegen \& de Jong 1993, 1994a,b,c; Groenewegen et al. 1995; van den Hoek 1997; Wagenhuber \& Groenewegen 1998; Marigo 1998, 2001, 2002; Marigo et al. 1996, 1998, 1999, 2002, 2003; Mowlavi 1999; Mouhcine \& Lançon 2002). We therefore do not need to describe the basics of such models in detail, but restrict our discussion to a brief description of how we calculate our particular models and then use them to determine the quantities of dust formed and the composition of the dust mixture.

\subsection{Evolution up to the TP-AGB}

As a lower limit of the mass spectrum of stars that we are interested in, we take $M_{1}=1 M_{\odot}$. Stars with a somewhat smaller mass (down to about $0.8 M_{\odot}$ ) also reach the thermally pulsing AGB (TP-AGB) phase during the lifetime of the galaxy, but their mass-return to the interstellar medium is much too small, as that they are significant contributors of freshly synthesized nuclei and dust to the ISM.

Evolutionary models of stars with initial masses from $1 M_{\odot}$ to $7 M_{\odot}$ and different metallicities from $Z=0.001$ to $Z=0.02$ were taken from Schaller et al. (1992), Schaerer et al. (1993), and Charbonnel et al. (1993). The models for low mass stars $\left(M_{*} \leq 2.2 M_{\odot}\right)$ from these sources end with He ignition on the tip of the RGB and do so for intermediate mass stars $\left(2.2 M_{\odot} \leq\right.$ $M_{*} \leq 7 M_{\odot}$ ) with the first thermal pulse.

For intermediate mass stars, the models already include the effects of the first and second "dredge-up" on the stellar surface abundance and the mass-loss on the RGB and during the E-AGB-evolution up to the first thermal pulse. They give the initial mass of the stars and the initial elemental abundances for the models at the base of the TP-AGB.

Low-mass stars suffer no second "dredge-up" on the early Asymptotic Giant Branch (E-AGB) such that one can take the element abundances in the envelope at the tip of the RGB as the envelope abundance at the begining of the TP-AGB phase. The total amount of mass lost up to the TP-AGB is taken as the difference between the masses of the last model on the tip of the RGB and the initial mass on the main sequence, since the mass-loss during the He core-burning phase and on the E-AGB is negligible for low-mass stars.

\subsection{Synthetic AGB-evolution}

For the evolution of the mass, luminosity, radius, and effective temperature of the stars on the TP-AGB, we closely follow the model calculations of Groenewegen \& de Jong (1993) and Marigo et al. (1996). The details can be found in these two papers.

The star is described by its total mass $M_{*}$ and its core mass $M_{\mathrm{c}}$. The core mass includes the He-shell (of about $0.01, \ldots, 0.02 M_{\odot}$ mass). The mass of the hydrogen rich envelope is

$M_{\mathrm{e}}=M_{*}-M_{\mathrm{c}}$.

All masses given in the following are in units of solar masses.

\subsubsection{Luminosity evolution}

The luminosity due to H-burning is given by a core-mass luminosity relation of the type

$L_{\mathrm{H}}=F\left(M_{\mathrm{c}}, M_{*}, Z_{\mathrm{CNO}, \mathrm{e}}, \mu_{\mathrm{e}}\right)$,

where

$\mu_{\mathrm{e}}=\frac{4}{5 X_{\mathrm{e}}+3-Z_{\mathrm{e}}}$

is the mean molecular weight of the envelope material and $Z_{\mathrm{CNO}, \mathrm{e}}$ the total abundance by mass of $\mathrm{C}, \mathrm{N}$, and $\mathrm{O}$ within the envelope. The evolution of the luminosity is determined by the evolution of the core and total mass and by the evolution of the composition of the envelope material. In our calculations we use the approximations given in Groenewegen \& de Jong (1993) for $L_{\mathrm{H}}$. The total luminosity $L_{*}$ is obtained by adding 
some corrections due to He-burning and gravitational contraction to $L_{\mathrm{H}}$ (see Groenewegen \& de Jong 1993).

The luminosity variations following a thermal pulse and corrections for the first pulses are also treated in the schematic approximation of Groenewegen \& de Jong (1993). We do not use the more accurate prescriptions given in Wagenhuber \& Groenewegen (1998) since then one has to follow the luminosity variations in much more detail, while for the simpler approximations of Groenewegen \& de Jong, the luminosity variations after a thermal pulse are represented by only three different states. This allows much bigger time steps and less frequent calculations of models for the dust shell. Since the whole model for dust production considered in this paper is still rather crude, the simple three-stage approximation for the post-flash luminosity variations of Groenewegen \& de Jong (1993) should be sufficient accurate.

\subsubsection{Core evolution in the inter-pulse phase}

The core growth in the period between thermal pulses is determined by hydrogen burning at the bottom of the envelope. It is described by

$\frac{\mathrm{d} M_{\mathrm{c}}}{\mathrm{d} t}=9.55 \times 10^{-12} \frac{L_{\mathrm{H}}}{X_{\mathrm{e}}}$.

Here $X_{\mathrm{e}}$ is the hydrogen abundance by mass in the envelope.

The time $T_{\text {ip }}$ (in years) between successive thermal pulses is given by (Groenewegen \& de Jong 1993)

$T_{\text {ip }}=\left\{\begin{array}{l}4.50\left(1.689-M_{\mathrm{c}}\right) \text { for } Z=0.02 \\ 4.59\left(1.644-M_{\mathrm{c}}\right) \text { for } Z=0.001 .\end{array}\right.$

For other metallicities, $T_{\text {ip }}$ is determined by linear interpolation between the two limit cases. $M_{\mathrm{c}}$ is the core mass at the beginning of each inter-pulse period. Corrections to the interpulse length for the first pulses are treated as in Groenewegen \& de Jong (1993).

\subsubsection{Envelope evolution in the inter-pulse phase}

The envelope mass decreases

- by hydrogen burning at the bottom of the envelope, and

- by massloss due to a stellar wind at the stellar surface.

The variation of envelope mass with time is therefore given by

$\frac{\mathrm{d} M_{\mathrm{e}}}{\mathrm{d} t}=-\frac{\mathrm{d} M_{\mathrm{c}}}{\mathrm{d} t}-\dot{M}_{\text {wind }}$.

The mass-loss rate $M_{\text {wind }}$ is discussed in Sect. 4.

The effective temperature $T_{\text {eff }}$ of the envelope is determined either by the approximations given in Wood (1990) or by the approximation given by Vassiliadis \& Wood (1993)

$\log T_{\text {eff }}=.234 \log M_{*}-.2 \log L_{*}-.116 \log \frac{Z}{0.02}+4.146$.

They relate $T_{\text {eff }}$ to the luminosity $L_{*}$, mass $M_{*}$, and metallicity $Z$. The parametrization (45) is applied only where it is necessary to achieve consistency with results of Gautschy (1999), see Sect. 4.2.1, since it requires that the stars have not yet started to significantly evolve off from the AGB. These approximations do not consider the dependence of the envelope properties on the $\mathrm{C} / \mathrm{O}$ abundance ratio, which has been shown by Marigo (2002) to be significant.

The radius $R_{*}$ of the envelope is determined from the standard relation

$4 \pi R_{*}^{2} \sigma T_{\mathrm{eff}}^{4}=L_{*}$.

\subsubsection{Evolution of the star in the inter-pulse phase}

Equations (42) and (44), together with the relations equating the luminosity and the wind mass-loss rate to the core and envelope masses and the element abundances $X_{\mathrm{e}}, Y_{\mathrm{e}}, Z_{\mathrm{e}}, Z_{\mathrm{CNO}, \mathrm{e}}$ are solved as a system of ordinary differential equations over the period $T_{\mathrm{ip}}$. They determine the run of $M_{\mathrm{c}}$ and $M_{\mathrm{e}}$ between thermal pulses. The integration is done with a fourth-order Runge-Kutta method. The stepwidth is chosen such that the accuracy of the integration per time step is on the order of $10^{-6}$.

At the first pulse, one needs the core and envelope mass as initial values in order to start the integrations. For intermediatemass stars, $M_{\mathrm{c}}$ is determined from the luminosity of the last model from the stellar evolution models (see Sect. 3.1) and the core mass-luminosity relation. For low-mass stars we calculate the core mass at the first pulse from the approximation Eq. (1) of Groenewegen \& de Jong (1993). Then $M_{\mathrm{e}}$ follows in both cases from $M_{\mathrm{c}}$ and the total mass.

\subsection{Dredge-up following a thermal pulse}

After a few thermal pulses, mixing processes begin to operate after each thermal pulse that carries nuclear processed material from the He-shell to the stellar surface. As a result the $\mathrm{He}, \mathrm{C}$, and $\mathrm{O}$ abundances of the envelope increase after each such mixing event. Since the abundance of $\mathrm{C}$ in the He shell material is significantly higher than the $\mathrm{O}$ abundance, the $\mathrm{C} / \mathrm{O}$ abundance ratio in the envelope increases after each third "dredge-up" episode and after a certain number of pulses, the star becomes a carbon star.

The third "dredge-up" is of basic importance for the nucleosynthesis of $\mathrm{C}$ and $\mathrm{N}$ in the galaxy, since the returned mass from AGB-stars is a major, or even the dominating, source for these elements (e.g. Thielemann 2002); and it is important for the dust production of AGB-stars, since the nature and quantities of dust produced in the stellar outflows critically depend on the $\mathrm{C} / \mathrm{O}$ abundance ratio.

The "dredge-up" episodes start if the core mass at a thermal pulse exceeds some critical value $M_{\mathrm{c}, \text { dredge }}^{\text {min }}$. If the lower boundary of the outer convection zone penetrates into the He shell during the after-pulse phase, part of the material of the He shell is mixed with envelope material. The corresponding mass is usually written as

$\Delta M_{\text {dredge }}=\lambda \Delta M_{\mathrm{c}}$,

where $\Delta M_{\mathrm{c}}$ is the increment of the core mass during the interpulse phase preceding the actual pulse. 
$M_{\mathrm{c}, \mathrm{dredge}}^{\min }$ and $\lambda$ are parameters that are empirically determined in the older prescriptions for third dredge up by fitting the results of synthetic AGB-evolution to the observed distribution of carbon stars in the Large Magellanic Cloud. Groenewegen \& de Jong (1993) give $M_{\mathrm{c}, \text { dredge }}^{\min }=0.58$ and $\lambda=0.75$, while Marigo et al. (1996) obtained $\lambda=0.65$. However, the assumption of a single value of $\lambda$ for all stellar masses and metallicities seems to be a somewhat strong simplification, and more recent models of synthetic AGB-evolution by Marigo et al. (2003) with different values of $\lambda$ for different stellar masses seem to give better results.

In the most recent determination of the "dredge-up" parameters $\lambda$ and $M_{\mathrm{c}, \text { dredge }}^{\min }$ by Karakas et al. (2002), the variation of $\lambda$ and $M_{\mathrm{c}, d r e d g e}^{\min }$ with stellar initial mass $M_{0}$ and metallicity has been determined by fitting results of model calculations of AGB evolution. The prescription to calculate

$M_{\mathrm{c}, \text { dredge }}^{\min }=M_{\mathrm{c}, \text { dredge }}^{\min }\left(M_{0}, Z\right)$

and the prescription for calculating

$\lambda=\lambda\left(M_{0}, Z, N\right)$,

where $N$ is the number of the pulse, are taken from Karakas et al. (2002).

After each pulse the core and envelope masses are reset as follows:

$M_{\mathrm{c}, \text { new }}=M_{\mathrm{c}, \text { old }}-\lambda \Delta M_{\mathrm{c}}$

$M_{\mathrm{e}, \text { new }}=M_{\mathrm{e}, \text { old }}+\lambda \Delta M_{\mathrm{c}}$,

where $M_{\mathrm{c}, \text { old }}$ and $M_{\mathrm{e} \text {,old }}$ denote the core and envelope mass at the end of the inter-pulse phase, while $M_{\mathrm{c} \text {,new }}$ and $M_{\mathrm{e}, \text { new }}$ serve as initial values for integrating Eqs. (42) and (44) during the next inter-pulse phase.

The abundances of some nuclei in the envelope change due to the mixture of the envelope with material from the He shell. The abundances after the "dredge-up" episode are

$X_{i, \text { new }}=\frac{X_{i, \text { old }} M_{\mathrm{e}, \text { old }}+X_{i, \text { shell }} \lambda \Delta M_{\mathrm{c}}}{M_{\mathrm{e}, \text { new }}}$.

Here $X_{i, \text { old }}$ is the abundance by mass of species $i$ in the envelope prior to "dredge-up", $X_{i, \text { new }}$ its abundance after "dredge-up", and $X_{i \text {,shell }}$ its abundance in the He shell material at the time of "dredge-up".

In this calculation we consider only the evolution of the elements $\mathrm{H}, \mathrm{He}, \mathrm{C}, \mathrm{N}$, and $\mathrm{O}$. For the $\mathrm{He}$ shell we assume the same composition with respect to the isotopes of these elements as Marigo et al. (1996):

$X^{4} \mathrm{He}$,shell $=0.76$

$X^{{ }^{12} \mathrm{C} \text {,shell }}=0.22$

$X_{{ }^{16} \mathrm{O}, \text { shell }}=0.02$.

The $X_{i \text {,shell }}$ of all other isotopes $i$ of these elements are assumed to be zero in the He shell. For all nuclei of the heavier elements we now assume that their abundances in the shell equal the one in the envelope and do not change by the "dredge-up" episodes, since changes of their abundances are of minor importance in the dust-formation problem.
It has been found that the "dredge-up" episodes end or become inefficient if the envelope mass is reduced below a certain minimum mass. Straniero et al. (1997) gave $M_{\mathrm{e}, \mathrm{dredge}}^{\mathrm{min}}=0.50$ as a limit. This seems to be roughly in accord with observations of the presence of Tc in the atmospheres of AGB stars (Lebzelter \& Hron 2003). In their set of full AGB evolutionary models Karakas et al. (2002), however, found that the lower limit for the envelope mass for the third "dredge-up" to occur must be below $0.2 M_{\odot}$. Also, calculations of more advanced evolutionary phases at the transition from AGB stars to White Dwarfs show that "dredge-up" is important even for rather small envelope masses (e.g. Blöcker 2001; Herwig 2001). Because the situation is unclear with respect to the existence of a lower limit to the envelope mass for this third dredge up to occur, we only present models with $M_{\mathrm{e}, \text { dredge }}^{\min }=0$.

\subsection{Hot bottom burning}

Our models account for the phenomenon of hot bottom burning in a very simplified way, by assuming that it occurs for stars with initial mass from $4 M_{\odot}$ upward, as long as the envelope mass exceeds the limit (Wagenhuber \& Groenewegen 1998)

$M_{\mathrm{e}, \mathrm{HBB}}^{\min }=M_{*}(0.7-5 Z)$.

It is then assumed that all $\mathrm{C}$ and $\mathrm{N}$ nuclei after a third "dredge-up" event are immediately converted into their equilibrium isotopic abundance within the $\mathrm{CN}$-cycle, for which we take

${ }^{15} \mathrm{~N} /{ }^{14} \mathrm{~N}=2.78 \times 10^{-5}$

${ }^{12} \mathrm{C} /{ }^{14} \mathrm{~N}=4.07 \times 10^{-2}$

${ }^{13} \mathrm{C} /{ }^{12} \mathrm{C}=0.25$

as typical for a temperature of $60 \times 10^{6} \mathrm{~K}$ (Clayton 1968). These assumptions are uncritical in the chemistry of dust formation, since the element mixture remains oxygen rich due to hot bottom burning and then the dust formation does not depend on the abundances of $\mathrm{C}$ and $\mathrm{N}$.

This treatment of hot bottom burning is over-simplified and does not account for an only partial conversion of $\mathrm{C}$ into ${ }^{14} \mathrm{~N}$ for stellar masses at the lower mass limit for hot bottom burning and for the excess of luminosity over that calculated from the simple core-mass luminosity relations we use. We thus overestimate both the evolution time during which the star remains an M-star and underestimate the mass-loss rate during this phase. To some extent, these effects should cancel in calculating the dust production rate.

\subsection{End of AGB evolution}

The end of AGB evolution is assumed to be reached either if the envelope mass decreases below $0.1 \Delta M_{\mathrm{c}}$ (Iben 1985) or if the core mass reaches the Chandrasekhar limit for explosion by carbon ignition in a degenerate core. Since our main interest is the dust production of AGB stars, we do not follow the very last phases of shell ejection at the tip of the AGB but stop the evolution calculation in most cases already if the shell mass decreases below $10^{-2} M_{\odot}$, since during the remaining AGB lifetime only negligible quantities of dust are formed. 


\section{Mass-loss by stellar winds}

For the purpose of calculating dust production in circumstellar dust shells, we cannot apply a single mass-loss prescription for all stellar types in the right upper part of the Hertzsprung-Russel Diagram, as in Blöcker (1995), as it has been done in most other calculations of synthetic AGB evolution. Since the properties of the outflows and the efficiency of dust production strongly depend on the two questions (i) whether the stars are variable or not, and (ii) if they are variable, how they depend on the nature of their variability, we have to consider the different cases separately.

\subsection{Mass-loss at low luminosities}

On the RGB and on the lower part of the AGB, the stars are non-variable. The mechanism driving those observed stellar winds and massloss of giants with moderate luminosities up to a few $10^{3} L_{\odot}$ and low effective temperatures is not known. The properties of the wind and, in particular, the mass-loss rate cannot be determined at present from of a physical theory by first principles. We therefore follow the common practice of using an empirically determined approximate fit formula to determine the mass-loss rate for such stars. The most popular is the Reimers formula (Reimers 1975; Dupree 1986; Dupree \& Reimers 1987) and variants of it, which approximate observed mass-loss rates of red giants by

$\dot{M}_{\text {Reim }}=\eta \cdot 4 \times 10^{-13} \frac{L_{*} R_{*}}{M_{*}}$,

where $M_{*}, L_{*}$, and $R_{*}$ are the mass, luminosity, and stellar radius (in solar units); and where $\dot{M}_{\text {Reim }}$ is in solar masses per year and $\eta$ a parameter used to adjust the fit formula to particular cases. For calculating the massloss of Red Giants, we use the value $\eta=0.86$ given by Groenewegen \& de Jong (1993). Lines of constant mass-loss rate according to Eq. (60) in the HR-diagram are shown in Fig. 2.

Some test calculations with the value of $\eta=1.4$ recommended by Kudritzki \& Reimers (1978) for intermediate mass stars are performed, but this does not significantly change the final results, except for stars from the upper end of the mass spectrum of intermediate mass stars, which lose a small but not completely neglible part of their mass on the early AGB.

\subsection{Mass-loss by variable stars}

AGB stars cross a region of the HR-diagram during their evolution where they are unstable against pulsations. Most AGB stars are Miras or Semiregular Variables of type SRa or SRb or irregular Long Period Variables of type Lb. Really non-variable stars apparently do not seem to exist in this part of the HR-diagram. By definition, Miras are pulsators with highamplitude magnitude variations in the visible spectral region, while the other types show only moderate-to-small amplitudes. The pulsation mode of these stars has long been unclear, but it has recently been found (Wood et al. 1999) that Miras are fundamental mode pulsators, while the other types pulsate in higher-order modes.

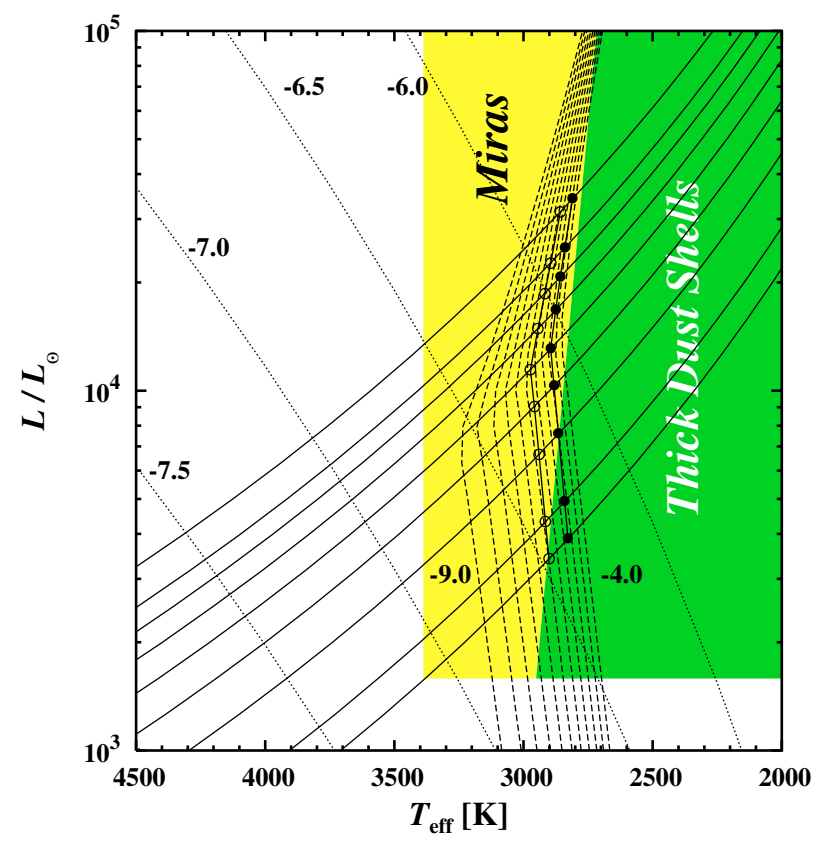

Fig. 2. Loci of stars along the AGB (full lines), lines of constant massloss rates (dotted and dashed lines), and the Mira instability strip (grey shaded area). The possible positions in the HR-diagram of AGB stars with different masses are shown for stellar masses of $0.8,1,1.5,2$, $2.5,3,3.5,4,5 M_{\odot}$, in this order from bottom to top. The lines of constant mass-loss rate, according to a Reimers-approximation (dotted lines), are labeled by $\log \dot{M}$ in units of $M_{\odot} \mathrm{yr}^{-1}$. The lines of constant mass-loss rate for pulsating Mira Variables in the approximation given by Vassiliadis \& Wood (1993) (dashed lines) are for mass-loss rates from $10^{-9}$ to $10^{-4} M_{\odot} \mathrm{yr}^{-1}$ in steps of $\log \dot{M}$ of 0.5 (from left to right). The thick line with circles indicates the transition line where the massloss due to pulsation exceeds the mass-loss as given by a Reimers approximation. The thick line with filled circles indicates the transition line to optically very thick dust shells.

It is known observationally that mass-loss rates are high only for Miras, which usually are also associated with significant circumstellar dust shells, while SRas, SRbs, and LpVs only exhibit small mass-loss rates and have only very thin dust shells, if any at all. From the point of view of dust production, the Miras are particulary important, because they are the most efficient dust factories on the AGB.

\subsubsection{The Mira instability strip}

The large-amplitude pulsations exhibited by the stars if they become Miras are generally thought to drive the observed massive massloss of such variable stars in the upper part of the AGB (cf. the review by Willson 2000). These stars appear in a limited region in the HR-diagram, where a second instability strip for stellar pulsations at low effective temperatures exists for fundamental and first-to-fourth overtone pulsators (e.g. Xiong et al. 1998).

The position of this Mira instability region in the HR-diagram was discussed in Wood \& Zarro (1981), Tuchman et al. (1979), Groenewegen \& de Jong (1994c), and Gautschy (1999). The most definite attempt to determine the region in the

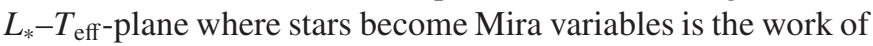


Gautschy (1999), on which we base our calculations. The effective temperature determined there is a kind of equilibrium temperature that corresponds to equilibrium states of the atmosphere. These temperatures are therefore just the appropriate ones that can be directly related to the effective temperatures calculated for the envelopes of synthetic AGB models. We use the results obtained by Gautschy (1999) for fundamental mode pulsators, since Miras are almost certainly fundamental mode pulsators (Wood et al. 1999).

Using data from Hughes \& Wood (1990) for Miras in the large Magellanic cloud, Gautschy found that the blue edge of the Mira instability strip is at $\log T_{\text {eff }}=3.53$. This edge seems to be rather well-defined, as can also be seen from Fig. 8 in Bergeat et al. (2002) for a sample of carbon stars. At the same time, the red edge seems to only slightly depend on luminosity. We approximate the results shown in Fig. 2 of Gautschy (1999) by

$\log T_{\text {eff }}=3.470-.020\left(\log L_{*} / L_{\odot}-3.200\right)$.

The lower border of the Mira instability strip is at $\log L_{*} / L_{\odot}=$ 3.20. In Fig. 2 of Gautschy (1999), an upper limit of the instability strip at $\log L_{*} / L_{\odot}=4.20$ also appears to exist, but this results from restricting the data used there for the inversion process to Miras with periods less than 500 day. Miras with longer periods are generally also more luminous, as can be seen nicely from Fig. 6 in Hughes \& Wood (1990). An upper limit on the order of $\log L_{*} / L_{\odot}=4.70$ seems to be indicated by this figure, but this is probably the upper limit to where stars from the upper range of initial masses of intermediate-mass stars leave the AGB and start to evolve towards White Dwarfs and not really the upper limit for the existence of the Mira instability. We do not impose an upper limit to the Mira instability strip in our calculations, because the position of such a possible limit is apparently not known at present and possibly does not exist.

The redwards edge, as shown in Gautschy (1999), refers to optically visible Miras. Variable stars with optically thick dust shells and pulsational properties like Miras are probably also located to the right of this border (see e.g. Fig. 9 of Bergeat et al. 2002).

For galactic Miras, Gautschy (1999) finds a location for the blue edge of the Mira instability strip at $\log T_{\text {eff }}=3.49$. The red edge is not well-defined because of the small number of data points, possibly it is located below $\log T_{\text {eff }}=3.43$. This would accord with the theoretical results of Xiong et al. (1998), who found a redwards shift of the Mira instability strip of galactic Miras compared to that of the Magellanic cloud, due to increasing metallicity. Xiong et al. (1998) predicted a shift by about $350 \mathrm{~K}$ towards higher temperatures if the metallicity is reduced from galactic metallicity to one half of it. This is roughly in accord with the empirical result of Gautschy (1999).

The lower border of the instability strip for galactic Miras also is not well-defined. From Fig. 7 of Gautschy (1999), we take $\log L_{*} / L_{\odot}=3.25$ as a crude estimate of this limit. For other metallicities we interpolate the borders of the Mira instability strip linearly in $Z$. The position of the Mira instability strip for $Z=0.008$ is shown in Fig. 2 .

The theoretical calculations of Xiong et al. (1998) also indicate that the instability strip is different not only for stars with different metallicities but also for different luminosities and masses. The empirically determined instability strip thus seems to be a superposition of different instability strips for stars with different masses and luminosities. Because of the lack of data, we cannot include such dependencies in our calculations and thus take the instability strip as defined above for all stellar types, except for the redwards border, which is discussed further below.

\subsubsection{Mass-loss prescription for Miras}

Mass-loss rates for Mira pulsators are determined by using the same prescriptions as Marigo et al. (1996), which are themselves the empirically determined relations of Vassiliadis \& Wood (1993). The pulsational period $P$ (in days) is calculated from

$\log P=-2.07+1.94 \log R_{\mathrm{e}} / R_{\odot}-0.90 \log M / M_{\odot}$,

with $R_{\mathrm{e}}$ and $M$ the stellar radius and mass, respectively. The mass-loss rate in units $M_{\odot} \mathrm{yr}^{-1}$ is determined from the empirical relation of Vassiliadis \& Wood (1993) for stars with mass $M<2.5 M_{\odot}$

$\log \dot{M}_{\text {Mira }}=-11.40+1.23 \times 10^{-2} P$

and

$$
\begin{aligned}
\log \dot{M}_{\text {Mira }}= & -11.40 \\
& +1.23 \times 10^{-2}\left(P-100\left(M / M_{\odot}-2.5\right)\right)
\end{aligned}
$$

for stars with mass $M \geq 2.5 M_{\odot}$. Lines with a constant massloss rate in the HR-diagram according to these prescriptions, Eqs. (63) or (64), are shown in Fig. 2 with dashed lines.

Given the stellar parameters $L_{*}$ and $M_{*}$ and $Z$, one calculates $T_{\text {eff }}$ from Eq. (45) or from the equations in Wood (1990) and then $\dot{M}$ from the above equations. Having fixed these parameters, wind models are calculated as described in Sect. 2. In Fig. 2 the filled dots show where the optical depth of the dust shell calculated with $\left\langle\kappa_{\mathrm{H}}\right\rangle$ for each of the stellar masses and an oxygen-rich element mixture becomes

$\tau_{\mathrm{H}}=\int_{R_{\mathrm{i}}}^{R_{\mathrm{a}}} \mathrm{d} r \rho\left\langle\kappa_{\mathrm{H}}\right\rangle=1$,

where $R_{\mathrm{i}}$ and $R_{\mathrm{a}}$ are the inner and outer boundaries of the dust shell. This defines the limit where the dust shells become optically very thick and the central stars become invisible. This limit is obviously identical to the red border of the Mira instability strip as found by Gautschy (1999). For this reason we assume that the red border does not correspond to the real border of the Mira instability strip, which must be located somewhere to the right of it. The true position of this border remains unclear. Since in our calculations of synthetic AGB evolution, the stars do not evolve very much to the right of the border where the dust shells become optically very thick, we assume that stars also to the right of this limit line in the HR-diagram remain Mira variables.

More detailed results of our calculation for the position of the limit line where $\tau_{\mathrm{H}}=1$ are shown in Table 2 and in 
Table 2. Models for oxygen-rich dust shells with an optical depth $\tau_{\mathrm{H}}=$ 1. These define the limit between optically visible Miras and heavily obscured objects.

\begin{tabular}{cccccc}
\hline \hline$M_{*}$ & $\begin{array}{c}L_{*} \\
M_{\odot}\end{array}$ & $\begin{array}{c}T_{\text {eff }} \\
\mathrm{K}\end{array}$ & $\begin{array}{c}\dot{M} \\
M_{\odot} \mathrm{yr}^{-1}\end{array}$ & $\begin{array}{c}P \\
\text { days }\end{array}$ & $\begin{array}{c}v_{\text {exp }} \\
\mathrm{km} \mathrm{s}^{-1}\end{array}$ \\
\hline 0.8 & 3890 & 2830 & $6.10 \times 10^{-6}$ & 503 & 6.0 \\
1.0 & 4940 & 2840 & $7.27 \times 10^{-6}$ & 509 & 6.4 \\
1.5 & 7610 & 2860 & $1.01 \times 10^{-5}$ & 521 & 7.3 \\
2.0 & 10340 & 2880 & $1.27 \times 10^{-5}$ & 528 & 7.9 \\
2.5 & 13120 & 2900 & $1.52 \times 10^{-5}$ & 535 & 8.4 \\
3.0 & 16810 & 2870 & $1.91 \times 10^{-5}$ & 593 & 9.3 \\
3.5 & 20790 & 2860 & $2.34 \times 10^{-5}$ & 650 & 10.1 \\
4.0 & 25030 & 2840 & $2.77 \times 10^{-5}$ & 706 & 10.8 \\
5.0 & 34270 & 2810 & $3.70 \times 10^{-5}$ & 817 & 12.0 \\
\hline
\end{tabular}

Fig. 2 (thick line with filled circles). The limit line $\tau_{\mathrm{H}}=1$ coincides quite well with the red limit (border between light grey and dark grey shaded areas in Fig. 2) for optically visible Miras (Eq. (61)) as estimated from the observational data of Gautschy (1999), if one considers the very approximate nature of the obervationally determined limit. The limit line $\tau_{\mathrm{H}}=1$ also corresponds to pulsation periods of about $P=500$ days, except for the highest masses. That the limit determined from our simplified wind model coincides with the observed limit for visible Miras as determined by Gautschy (1999) and with the limit for transition to objects with massive dust shells at $P \approx 500$ days as found by Vassiliadis \& Wood (1993) supports our assumption that the kind of simplified wind model combined with our model for calculating the dust formation yields results of sufficient accuracy to determine the dust production rate of AGB stars. Only the expansion velocity is somewhat low compared to observed outflow velocities. This may either result from our neglect of the effect of shocks or from the opacity of silicates used in the model (see Sect. 2.1.4) being slightly too small.

For models with $\tau_{\mathrm{H}}>1$, we follow the assumptions of Vassiliadis \& Wood (1993) and Marigo et al. (1996) and determine the mass loss-rate in the superwind phase from the approximation

$\dot{M}_{\mathrm{SW}}=2.1 \times 10^{-8} \frac{L / L_{\odot}}{v_{\infty}\left[\mathrm{km} \mathrm{s}^{-1}\right]}$,

where $v_{\infty}$ is the terminal velocity of the wind. This velocity is also determined from the approximation given in Vassiliadis \& Wood (1993) as

$v_{*}=-13.50+5.60 \times 10^{-2} P \mathrm{~km} \mathrm{~s}^{-1}$

and then

$v_{\infty}=\min \left(\max \left(v_{*}, 3 \mathrm{~km} \mathrm{~s}^{-1}\right), 15 \mathrm{~km} \mathrm{~s}^{-1}\right)$.

For carbon-rich stars an improved approximation for $\dot{M}$ has been proposed by Wachter et al. (2002), which is however restricted to carbon Miras. It cannot be applied to all chemical types like the relations of Vassiliadis \& Wood (1993) that are determined from observations of all chemical types. For this reason we do not apply the improved prescription for carbon stars so as to have a consistent description of massloss for all chemical types.

\subsubsection{The $\mathrm{SRa}, \mathrm{SRb}$, and $\mathrm{Lb}$ variables}

Outside the Mira instability strip, the AGB stars all seem to be small-amplitude pulsators. These variables of type SRa, $\mathrm{SRb}$, and Lb pulsate in higher-order pulsational modes (Wood et al. 1999). They have low observed mass-loss rates, almost all below $10^{-7} M_{\odot} \mathrm{yr}^{-1}$, and low expansion velocities (cf. Kerschbaum et al. 1996; Kerschbaum \& Olofson 1998, 1999; Kerschbaum 1999; Olofson et al. 2002). During the rather short period of AGB evolution of at most of some $10^{6} \mathrm{yr}$ (e.g. Groenewegen et al. 1995), they do not lose much mass and, since they are inefficient dust factories, they do not significantly contribute to the total dust production of AGB stars. For determining their mass-loss rates, we apply the parametrization (60), which results in mass-loss rates as low as observed mass-loss rates.

\subsubsection{Short-period Miras}

Mira variables tend to have very low mass-loss rates if their pulsational period is less than about $P=300$ days. If the massloss rate $\dot{M}_{\text {Mira }}$ calculated from Eqs. (63) or (64) is less than the mass-loss rate $\dot{M}_{\text {Reim }}$ according to Eq. (60), the latter result is used for $\dot{M}$; i.e. we assume that the more efficient of the two processes is responsible for the stellar outflow.

The transition line between the two cases is indicated in Fig. 2 by circles. It roughly corresponds to a pulsational period of about 300 days. The transition occurs inside the Mira instability strip as determined by Gautschy (1999), which means that Miras to the left of the transition line, which have rather short pulsational periods, are assumed to lose mass with a rate given by Eq. (60), while Miras located to the right of the transition line, which have longer pulsation periods, are assumed to lose mass at a rate as given by Eqs. (63), (64) or Eq. (66).

\section{Model calculation}

\subsection{AGB models}

For a set of initial stellar masses, we evolve synthetic AGB models by the prescriptions outlined in Sects. 3 and 4 until nearly complete loss of their envelope. We stop the integration once the envelope mass decreases below $10^{-2} M_{\odot}$. At each step in the calculations we determine whether the star falls into the Mira instability region or not.

Case 1: if the star is not a Mira, the mass-loss rate is set to the value $\dot{M}_{\text {Reim }}$ given by Eq. (60), and a constant outflow velocity model for the dust shell is calculated, cf. Sect. 2.6. This provides the properties of the dust shell, from which we need the final degree of condensation, in particular, at large distances for the different dust species, in order to determine the amount of dust produced by the star during its AGB evolution.

If the mass-loss rate $\dot{M}_{\text {Reim }}$ is very low - we choose as a limit $\dot{M}=3 \times 10^{-7} M_{\odot} \mathrm{yr}^{-1}$ - the dust production is 


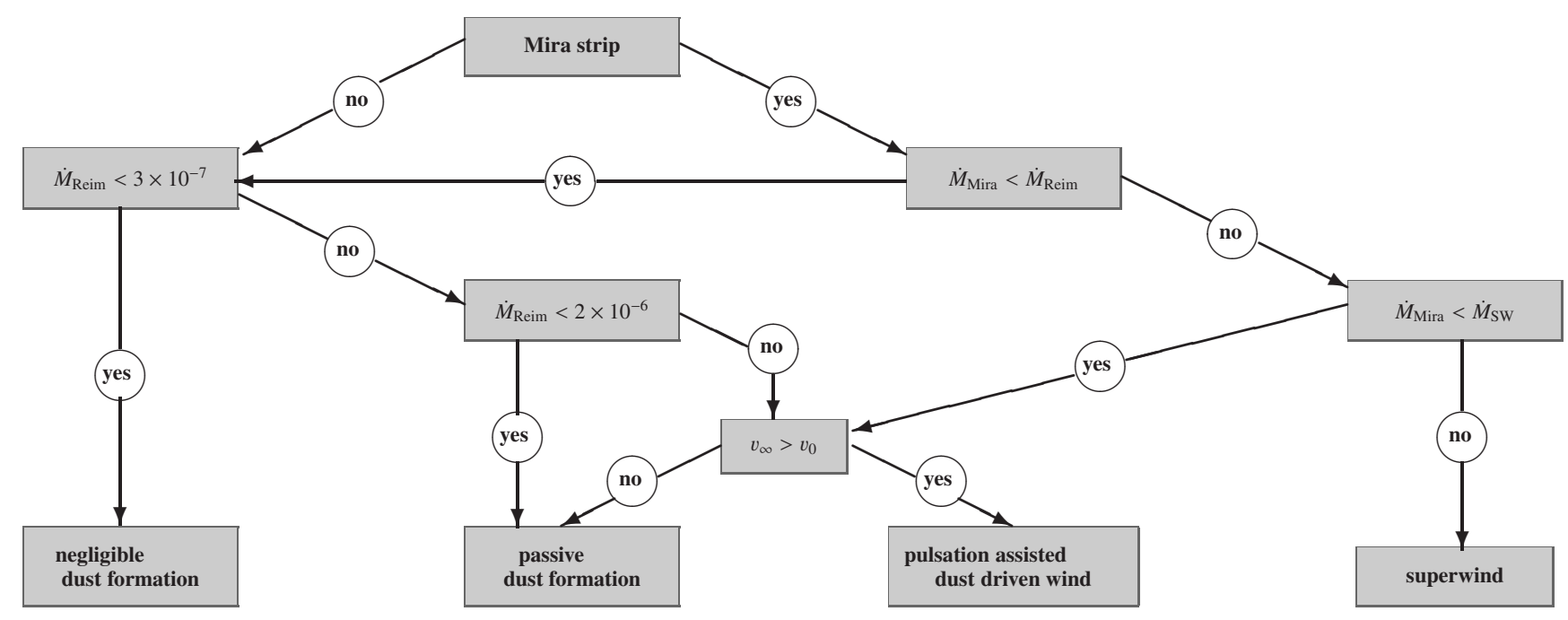

Fig. 3. Decision tree for the different cases of mass-loss prescriptions.

completely neglected, and we do not calculate a wind model at all but simply assume that no dust is formed.

For intermediate mass-loss rates $3 \times 10^{-7} \leq \dot{M}_{\text {Reim }} \leq 2 \times$ $10^{-6} M_{\odot} \mathrm{yr}^{-1}$, we assume that dust condensation is not efficient enough to drive the outflow. The outflow is assumed to result from some other process, and dust is only formed passively in the wind. In this case we calculate a constant outflow velocity dust shell model with an expansion velocity of $v_{\text {exp }}=5 \mathrm{~km} \mathrm{~s}^{-1}$. This expansion velocity is typical of low massloss rate stars (cf. Groenewegen et al. 1998; Kerschbaum et al. 1996; Kerschbaum \& Olofson 1999; Schöier \& Olofson 2001; Winters et al. 2003).

For higher mass-loss rates $\dot{M}_{\text {Reim }}>2 \times 10^{-6} M_{\odot} \mathrm{yr}^{-1}$, we assume that radiation pressure on dust suffices to drive the outflowing gas to supersonic outflow velocities. We calculate a dust-driven wind model and assume an initial velocity of $v_{\text {exp }}=2 \mathrm{~km} \mathrm{~s}^{-1}$ at the onset of dust formation. We check whether the final velocity $v_{\infty}$ is in fact significantly increased by radiation pressure over its initial value $v_{0}$. This check is necessary since even for rather high mass-loss rates, but luminosities below $1 \times 10^{4} L_{\odot}$, it may happen for $\mathrm{C} / \mathrm{O}$ abundance ratios between about 0.85 and 1.1 that insufficient silicate or carbon dust is formed to provide the necessary opacity in order to drive a wind. If the dust mixture is dominated by iron or iron alloy dust grains, their opacity is insufficient for driving a wind for luminosities below $L \approx 10^{4} L_{\odot}$, cf. Fig. 1 .

If the velocity increase due to dust formation is insignificant, the wind is driven by a different mechanism, for instance, by pulsation alone (Bowen 1988), so we calculate a constant outflow velocity model for the dust shell with an expansion velocity of $v_{\exp }=5 \mathrm{~km} \mathrm{~s}^{-1}$. If the velocity increase is significant, then we use the dust driven wind model. As criterion we use a $50 \%$ velocity increase. This arbitrary choice is uncritical, since the calculated models above $\dot{M} \approx 2 \times 10^{-6} M_{\odot} \mathrm{yr}^{-1}$ usually either fail to produce sufficient opacity or have highly supersonic outflow velocities. In our calculations the case of the wind being driven by radiation pressure on dust is apparently encountered only for Miras, but not for non-Miras.
Case 2: for Miras we check whether the mass-loss rate $\dot{M}_{\text {Reim }}$ calculated from Eq. (60) or $\dot{M}_{\text {Mira }}$ from Eqs. (63) or (64) is the higher one. If the prescription Eq. (60) predicts the higher mass-loss rate, we proceed as in the case of non-Miras.

In the second case of $\dot{M}_{\text {Mira }}>M_{\text {Reim, }}$, we use the massloss prescription $\dot{M}_{\text {Mira }}$ of Vassiliadis \& Wood (1993), Eqs. (63) or (64). The border between the two cases occurs at a pulsational period of about $300 \mathrm{~d}$ and the mass-loss rate then usually exceeds $\dot{M}=2 \times 10^{-6} M_{\odot} \mathrm{yr}^{-1}$, cf. Fig. 2 .

If $\dot{M}_{\text {Mira }}$ is less than $\dot{M}_{\text {SW }}$ calculated from Eq. (66), one has to take $\dot{M}_{\text {Mira }}$ as the mass-loss rate of the star, otherwise $\dot{M}_{\text {SW }}$. We calculate a dust-driven wind model with an initial velocity of $v_{0}=2 \mathrm{~km} \mathrm{~s}^{-1}$ at the onset of dust formation. Again, we check whether the calculated wind model leads to a significant velocity increase over the initial velocity and calculate a new constant outflow velocity model with $v_{\exp }=5 \mathrm{~km} \mathrm{~s}^{-1}$, if the velocity increase is insignificant. Usually, however, the wind turns out to be driven by radiation pressure. The way how the mass-loss rate is determined is illustrated in Fig. 3.

In this way at each integration step of the synthetic AGB evolution we calculate a dust model, which amounts to at least 12 model calculations for the dust shell per time step of the AGB evolution, since we use a fourth-order Runge-Kutta method with time-step control. The integration error per step of the synthetic AGB evolution is held at the order of $10^{-6}$. The considerable number of wind models that have to be calculated during the course of the AGB evolution motivated us to use a rather simple model both for the dust condensation and the AGB evolution. The computational time requirements, however, finally turned out not to be as stringent as we initially suspected and considerable improvements of the synthetic AGB evolution model and the wind model seem possible in future calculations.

The main output of the models for our present purposes are the degrees of condensation of the different dust species, but other quantities can be calculated as well, such as infrared spectra by an integrated radiative transfer code. 


\subsection{Dust production by AGB-stars}

The dust mass produced during the evolution on the AGB is calculated for the main dust species, which are:

- olivine-, pyroxene-, and quartz-type silicate dust grains and metallic iron dust grains during the M-star phase of the AGB evolution;

- metallic iron and quartz-type dust grains during the S-star phase of the AGB evolution; and

- carbon, $\mathrm{SiC}$, and metallic iron dust grains during the $\mathrm{C}$-star phase of the AGB evolution.

This is most easily done by calculating the mass $M_{i}(t)$ formed up to the instant $t$ from the begin of the thermally pulsing AGB evolution for each dust species $i$. This calculation is done separately for the three chemically different evolutionary stages on the AGB where stars are M-, S-, or C-stars.

\subsubsection{M-stars}

Since at present it does not seems possible to observationally discriminate between different silicate compounds with different composition for interstellar dust, we sum up our results for the three silicate compounds considered in our calculation and calculate the total production rate of silicate dust. The total mass $M_{\mathrm{sil}, \mathrm{M}}(t)$ of the silicate dust mixture formed up to instant $t$ during the M-star phase is determined by the equation

$\frac{\mathrm{d} M_{\mathrm{sil}, \mathrm{M}}}{\mathrm{d} t}=\dot{M} X_{\mathrm{Si}} \frac{A_{\mathrm{sil}}}{A_{\mathrm{Si}}} f_{\text {sil }}$,

where $\dot{M}$ is the mass-loss due to the stellar wind as calculated by the synthetic AGB evolution models, and $X_{\mathrm{Si}}$ is the element abundance of $\mathrm{Si}$ in the wind material by mass. Since $\mathrm{Si}$ is not subject to significant nuclear processing prior to or during AGB evolution, $X_{\mathrm{Si}}$ equals the initial abundance of $\mathrm{Si}$ in the material from which the star was formed. Finally, $A_{\mathrm{Si}}$ is the atomic weight of $\mathrm{Si}$.

The quantity $f_{\text {sil }}$ is the fraction of the element Si condensed into silicate dust grains at the outer radius of the wind models as discussed in Sect. 2. It is given by the degrees of condensation of the individual silicate dust components as

$f_{\text {sil }}=f_{\mathrm{ol}}+f_{\mathrm{py}}+f_{\mathrm{qu}}$.

The quantity $A_{\text {sil }}$ is the effective molecular weight of the silicate dust mixture which is given by the individual atomic weights of the silicate dust components as follows:

$$
\begin{aligned}
A_{\text {sil }}= & {\left[\left(x_{\mathrm{ol}} A_{\mathrm{fo}}+\left(1-x_{\mathrm{ol}}\right) A_{\mathrm{fa}}\right) f_{\mathrm{ol}}\right.} \\
& +\left(x_{\mathrm{py}} A_{\mathrm{en}}+\left(1-x_{\mathrm{py}}\right) A_{\mathrm{fs}}\right) f_{\mathrm{py}} \\
& \left.+A_{\mathrm{qu}} f_{\mathrm{qu}}\right] f_{\mathrm{sil}}^{-1},
\end{aligned}
$$

where $A_{\mathrm{fo}}$ and $A_{\mathrm{fa}}$ are the atomic weights of forsterite $\mathrm{Mg}_{2} \mathrm{SiO}_{4}$ and fayalite $\mathrm{Fe}_{2} \mathrm{SiO}_{4}$, respectively, which are the end members of the solid solution series of olivine. $A_{\mathrm{en}}$ and $A_{\mathrm{fs}}$ then are the atomic weights of enstatite $\mathrm{MgSiO}_{3}$ and ferrosilite $\mathrm{FeSiO}_{3}$, which are the end members of the solid solution series of pyroxene, an finally $A_{\text {qu }}$ is the atomic weight of quartz.
Analogously, the mass $M_{\text {iro,M }}(t)$ of the metallic iron dust formed up to instant $t$ is determined by the equation

$$
\frac{\mathrm{d} M_{\text {iro, } \mathrm{M}}}{\mathrm{d} t}=\dot{M} X_{\mathrm{Fe}} \frac{A_{\text {iro }}}{A_{\mathrm{Fe}}} f_{\text {iro }} \text {. }
$$

The meanings of the terms are analogous to the preceding case. The abundance of $\mathrm{Fe}$ is also not substantially changed during AGB evolution, though a small fraction of it is consumed by the formation of $s$-process elements, and its abundance is slightly reduced in the material "dredged-up" by the third "dredge-up" episodes. The quantity $X_{\mathrm{Fe}}$ can simply be approximated by the initial Fe abundance. The fraction of the element Fe condensed into solid iron is $f_{\text {iro }}$, as discussed in Sect. 2.

During the subsequent S-star and C-star phase, we put

$\frac{\mathrm{d} M_{\text {sil,M }}}{\mathrm{d} t}=\frac{\mathrm{d} M_{\text {iro,M }}}{\mathrm{d} t}=0$,

since $M_{\mathrm{sil}, \mathrm{M}}$ and $M_{\mathrm{iro}, \mathrm{M}}$ denote the silicate and iron-dust mass formed in the M-star phase, and $M_{\mathrm{sil}, \mathrm{M}}$ and $M_{\mathrm{iro}, \mathrm{M}}$ by definition do not change in the other evolutionary stages.

The equations for $M_{\text {sil,M }}$ and $M_{\text {iro,M }}$ are solved with initial conditions

$M_{\mathrm{sil}, \mathrm{M}}=M_{\mathrm{iro}, \mathrm{M}}=0 \quad$ at $t=0$

simultaneously with the equations for the synthetic AGBevolution and yield the run of $M_{\mathrm{sil}, \mathrm{M}}(t)$ and $M_{\mathrm{iro}, \mathrm{M}}(t)$ during the whole AGB-evolution.

\subsubsection{S-stars}

During the S-star phase, Fe condenses into pure solid Fe or into $\mathrm{FeSi}$, which are not distinguished in this calculation. The mass $M_{\text {iro,S }}(t)$ of the iron dust formed up to instant $t$ during the $\mathrm{S}$-star phase is determined by the equation

$\frac{\mathrm{d} M_{\mathrm{iro}, \mathrm{S}}}{\mathrm{d} t}=\dot{M} X_{\mathrm{Fe}} \frac{A_{\text {iro }}}{A_{\mathrm{Fe}}} f_{\text {iro }}$,

where $f_{\text {iro }}$ is the degree of condensation of the element Fe into iron dust during the S-star phase. The mass $M_{\mathrm{sil}, \mathrm{S}}(t)$ of the silicate dust formed up to instant $t$ during the S-star phase is determined by the equation

$\frac{\mathrm{d} M_{\mathrm{sil}, \mathrm{S}}}{\mathrm{d} t}=\dot{M} X_{\mathrm{Si}} \frac{A_{\text {sil }}}{A_{\mathrm{Si}}} f_{\text {sil }}$,

where $f_{\text {sil }}$ is the degree of condensation of the element Si into silicate dust, which during the S-star phase is assumed to be essentially quartz-type dust, see Sect. 2 . All other terms in the two equations have the same meaning as above. The element abundances $X_{\mathrm{Fe}}$ and $X_{\mathrm{Si}}$ are given by their initial values on the main sequence, since the abundances of $\mathrm{Fe}$ and $\mathrm{Si}$ do not change during AGB evolution.

The equations for $M_{\text {iro,S }}$ and $M_{\text {sil,S }}$ are solved with initial conditions

$M_{\mathrm{iro}, \mathrm{S}}=M_{\mathrm{sil}, \mathrm{S}}=0 \quad$ at $t=0$

and during the preceding M-star and subsequent C-star phase of the AGB evolution we put

$\frac{\mathrm{d} M_{\mathrm{iro}, \mathrm{S}}}{\mathrm{d} t}=\frac{\mathrm{d} M_{\mathrm{sil}, \mathrm{S}}}{\mathrm{d} t}=0$ 
The C/O abundance ratio defining the limit between the S-star and the M-star phase is discussed in Sect. A.1.

\subsubsection{C-stars}

The masses $M_{\text {iro, } \mathrm{C}}(t)$ and $M_{\mathrm{sic}, \mathrm{C}}(t)$ of the iron dust and silicon carbide dust formed up to instant $t$ during the C-star phase are determined by the equations

$\begin{aligned} \frac{\mathrm{d} M_{\text {iro, } \mathrm{C}}}{\mathrm{d} t} & =\dot{M} X_{\mathrm{Fe}} \frac{A_{\text {iro }}}{A_{\mathrm{Fe}}} f_{\text {iro }} \\ \frac{\mathrm{d} M_{\mathrm{sic}, \mathrm{C}}}{\mathrm{d} t} & =\dot{M} X_{\mathrm{Si}} \frac{A_{\mathrm{sic}}}{A_{\mathrm{Si}}} f_{\text {sic }},\end{aligned}$

where $f_{\text {iro }}$ and $f_{\text {sic }}$ are the degrees of condensation of the elements Fe and Si into iron dust and silicon carbide dust, respectively, during the $\mathrm{C}$-star phase, as discussed in Sect. 2. The element abundances $X_{\mathrm{Fe}}$ and $X_{\mathrm{Si}}$ are given by their initial values on the main sequence, since the abundances of $\mathrm{Fe}$ and $\mathrm{Si}$ do not change during AGB evolution.

The mass $M_{\mathrm{car}, \mathrm{C}}(t)$ of the carbon dust formed up to instant $t$ during the $\mathrm{C}$-star phase is determined by the equation

$\frac{\mathrm{d} M_{\mathrm{car}, \mathrm{C}}}{\mathrm{d} t}=\dot{M} X_{\mathrm{C}} f_{\mathrm{car}}$,

where $f_{\text {car }}$ is the degree of condensation of the element $\mathrm{C}$ into carbon dust during the C-star phase as discussed in Sect. 2. No ratio of a molecular to an atomic weight enters into this equation, since for solid carbon they are identical. The abundance $X_{\mathrm{C}}$ considerably changes by the third "dredge-up" episodes during the evolution on the AGB. Here we have to use the actual value of $X_{\mathrm{C}}$ as calculated by the synthetic AGB evolution models.

The equations are subject to the initial conditions

$M_{\text {iro, } \mathrm{C}}=M_{\mathrm{sic}, \mathrm{C}}=M_{\mathrm{car}, \mathrm{C}}=0 \quad$ at $t=0$

and during the preceding M-star and S-star phase of the AGB evolution we put

$\frac{\mathrm{d} M_{\mathrm{iro}, \mathrm{C}}}{\mathrm{d} t}=\frac{\mathrm{d} M_{\mathrm{sic}, \mathrm{C}}}{\mathrm{d} t}=\frac{\mathrm{d} M_{\mathrm{car}, \mathrm{C}}}{\mathrm{d} t}=0$.

The limit between the S-star and the C-star phase is taken to be $\epsilon_{\mathrm{C}} / \epsilon_{\mathrm{O}}=1.0$.

\section{Results}

We have calculated grids of synthetic AGB evolution models in the mass-region $1, \ldots, 7 M_{\odot}$ and for metallicities $Z=0.04$, $Z=0.02, Z=0.008, Z=0.004$, and $Z=0.001$, in order to determine the dust input into the interstellar medium by AGB stars. The initial models at the onset of thermal pulses are taken from the library of the stellar evolution models of Schaller et al. (1992), Schaerer et al. (1993), and Charbonnel et al. (1993). From these models we also take the mass lost on the RGB and E-AGB. Element abundances are solar system abundances from Table 1, scaled with metallicity and (for $\mathrm{He}$, $\mathrm{C}, \mathrm{N}, \mathrm{O}$ ) with the abundance changes between main sequence and the onset of thermal pulsing taken from the stellar evolution models.
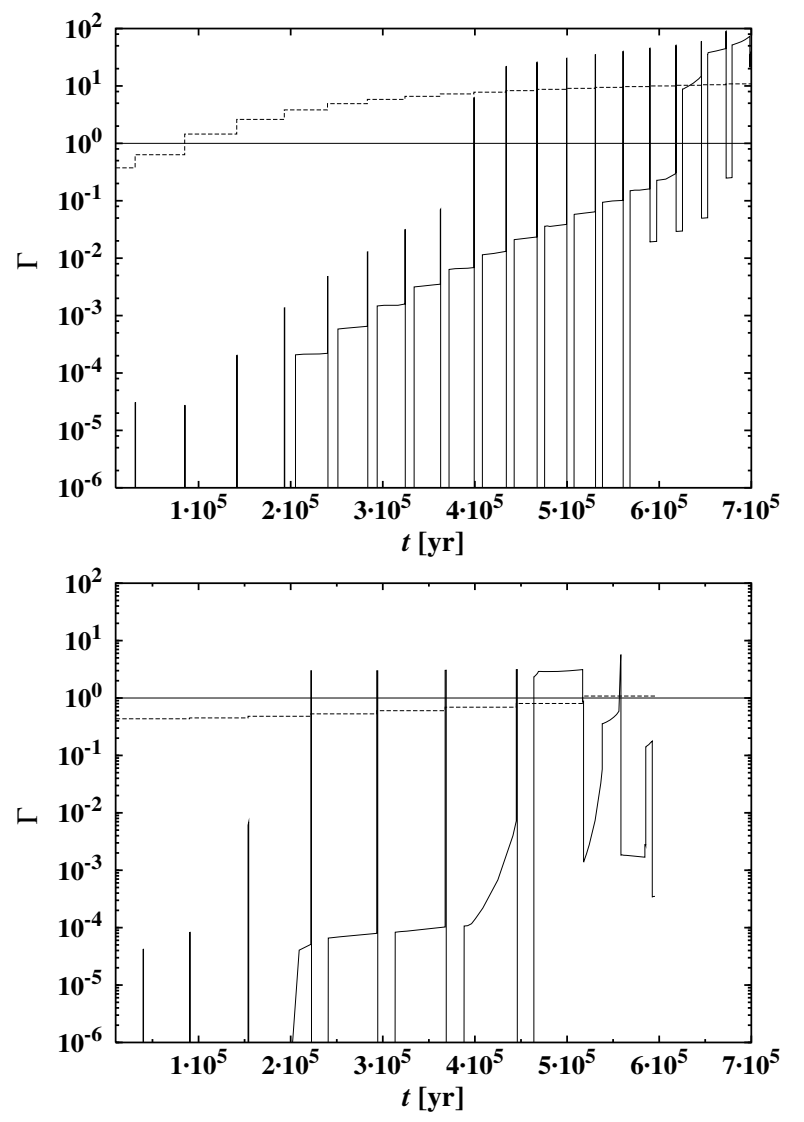

Fig. 4. Temporal variation of the ratio $\Gamma$ of radiation pressure to gravitational attraction of the star (full line) and the $\mathrm{C} / \mathrm{O}$ abundance ratio (dashed line) for a star with an initial mass of $2 M_{\odot}$ and metallicities of $Z=0.001$ (left part) and $Z=0.02$ (right part). The zero point of the time scale is at the first thermal pulse.

\subsection{Evolution of a $2 M_{\odot}$ star on the $A G B$}

As an example of results obtained for the dust formation on the $\mathrm{AGB}$, we consider the evolution of a star with an initial mass of $2 M_{\odot}$ and two different metallicities, a Pop I star with $Z=0.02$ and a Pop II star with $Z=0.001$.

Figure 4 shows the evolution of the ratio $\Gamma$ of outward directed radiation pressure on dust grains to inwardly directed gravitational attraction by the star, as defined by Eq. (2). For the figure, $\Gamma$ is determined by using the final degrees of condensation in the outflow for calculating $\kappa_{\mathrm{H}}$ according to Eq. (9). The outflow from the star is driven by radiation pressure on dust only during phases where $\Gamma>1$. During most of the evolution along the AGB, this requirement is met only during the short luminosity spikes following a thermal pulse. Only at the end of the AGB evolution, radiation pressure on dust starts to exceed the gravitational attraction by the star during a significant part of the inter-pulse phase, and the star enters the superwind phase with massive massloss by a dust driven-wind. Only for short phases during the luminosity dip following a thermal pulse or if and when the star happens to leave the Mira instability strip, does one have brief periods with $\Gamma<1$ also during the superwind phase.

The evolution of the $\mathrm{C} / \mathrm{O}$ abundance ratio is quite different for Pop I metallicity and for low metallicity. Due to the 

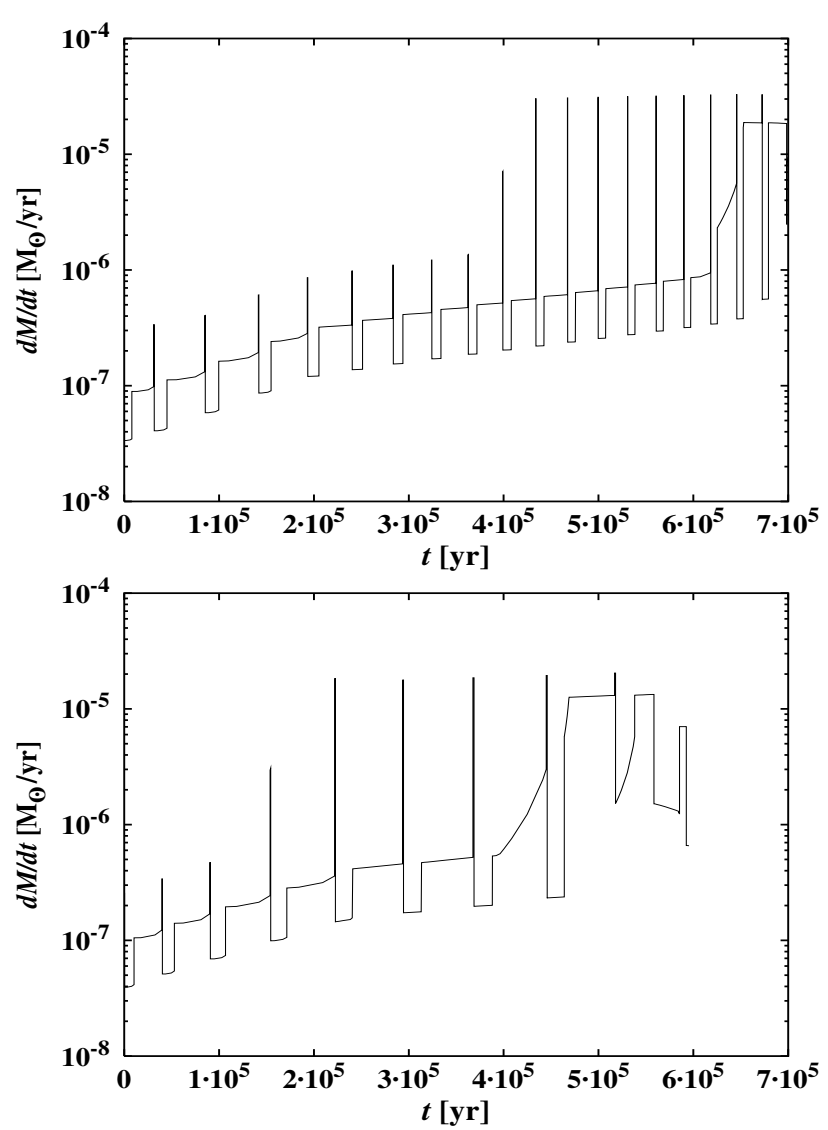

Fig. 5. Temporal variation of the mass-loss rate $\dot{M}$ for a star with an initial mass of $2 M_{\odot}$ and metallicities of $Z=0.001$ (left part) and $Z=0.02$ (right part). The zero point of the time scale is at the first thermal pulse.

low initial abundance of $\mathrm{C}$ and $\mathrm{O}$ in the envelope at the onset of thermal pulsing of the $Z=0.001$ model, the envelope becomes carbon rich after a few pulses, while after a number of additional pulses it approaches the $\mathrm{C} / \mathrm{O}$ abundance ratio of $\approx 10$ defined by the ratio of the abundances Eqs. (54) and (55) of $\mathrm{C}$ and $\mathrm{O}$ assumed for the He shell. During most of the time on the thermally pulsing AGB, the $Z=0.001$ model is a C-star. The S-star phase is skipped. For the Pop I model star, the $\mathrm{C} / \mathrm{O}$ abundance ratio increases more slowly due to the higher initial $\mathrm{C}$ and $\mathrm{O}$ content of the envelope. In this case the S-star phase is also skipped. Our definition of the S-star phase, however, is restricted to the phase of peculiar dust formation (see Sect. A.1). Spectroscopically, the star would appear for one inter-pulse phase as S-star, but the dust formed would be quite normal in this case.

Figure 5 shows the evolution of the mass loss-rates for both models. The mass-loss rates are rather low during most time of the evolution on the thermally pulsing AGB, but the star shows massive mass-loss episodes during the brief periods of enhanced luminosity following a thermal pulse. The superwind phase with very high mass-loss commences at the end of the evolution, if the luminosity has reached about $10^{4} L_{\odot}$ and the envelope mass has already strongly decreased. Evolution of the massloss of our $2 M_{\odot}$ Pop I model is similar to the one shown in Figs. 7 and 8 of Schröder et al. (1999) for their 1.85

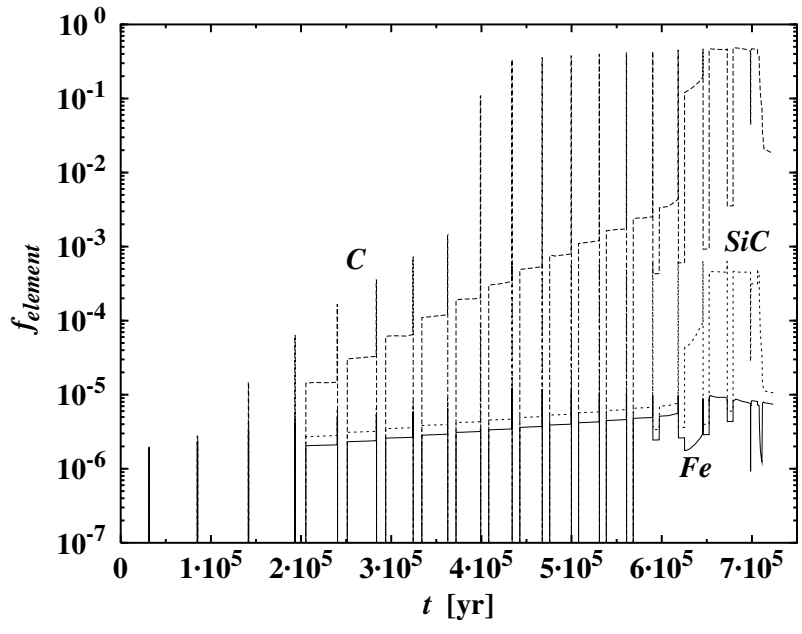

Fig. 6. Temporal evolution of the degree of condensation of the key elements for the main dust species during AGB evolution. Full line: degree of condensation of $\mathrm{C}$ in carbon dust. Dashed line: degree of condensation of $\mathrm{Si}$ into $\mathrm{SiC}$ dust grains. Dotted line: degree of condensation of $\mathrm{Fe}$ in metallic iron (or FeSi) grains. Model with initial mass $2 M_{\odot}$ and a metallicity of $Z=0.001$. The zero point of the time scale is at the first thermal pulse.

and $2.25 M_{\odot}$ models calculated with a stellar evolution code, though there are significant differences due to our schematic representation of the luminosity variations and our different prescriptions for the mass-loss rate.

Figure 6 shows the variation of the degree of condensation of the key elements into the different dust species for the model with $Z=0.001$. Dust condenses only in the evolutionary phase where the star is already a C-star. As can be seen, only carbon dust is formed in significant amounts. Other dust components are essentially absent. Figures 7 and 8 show the variation of the degree of condensations of the different dust species for the $Z=0.02$ model. Silicate dust is only formed (i) during the short high-mass-loss episodes after a thermal pulse; and (ii) for one interpulse period. In the subsequent "dredge-up" event the star switches to a carbon-rich composition and from then on forms significant amounts of carbon dust and $\mathrm{SiC}$.

Figure 9 shows the cumulative dust production over time during the three different phases where stars appear as M-stars, S-stars, or C-stars. During most of the time very little or no dust is produced during the inter-pulse periods. Some dust is formed during the brief luminosity spikes following a thermal pulse. Only during the last three inter-pulse phases does the mass-loss rate become sufficiently high for substantial amounts of dust to form and the wind to finally become driven by dust formation.

The mass-loss and dust production history shown in Figs. 4 to 9 should only be considered as giving a rough impression of how these quantities evolve as the star evolves up the TP-AGB. The details suffer from the rather crude approximation of the luminosity variations after a thermal pulse and probably will change, if this is treated more realistically. 

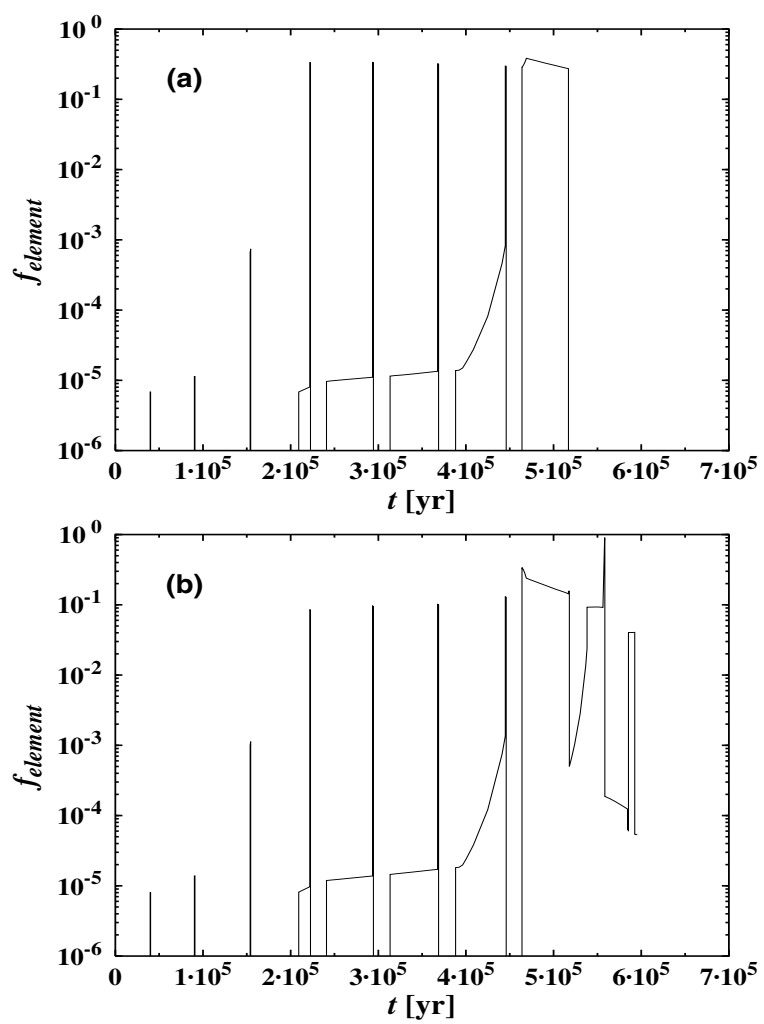

Fig. 7. Temporal evolution of the degree of condensation of the key elements for the main dust species during AGB evolution. a) Degree of condensation of Si in silicate dust species. b) Degree of condensation of $\mathrm{Fe}$ in metallic iron (or FeSi) grains. Model with initial mass $2 M_{\odot}$ and a metallicity of $Z=0.02$. The zero point of the time scale is at the first thermal pulse.
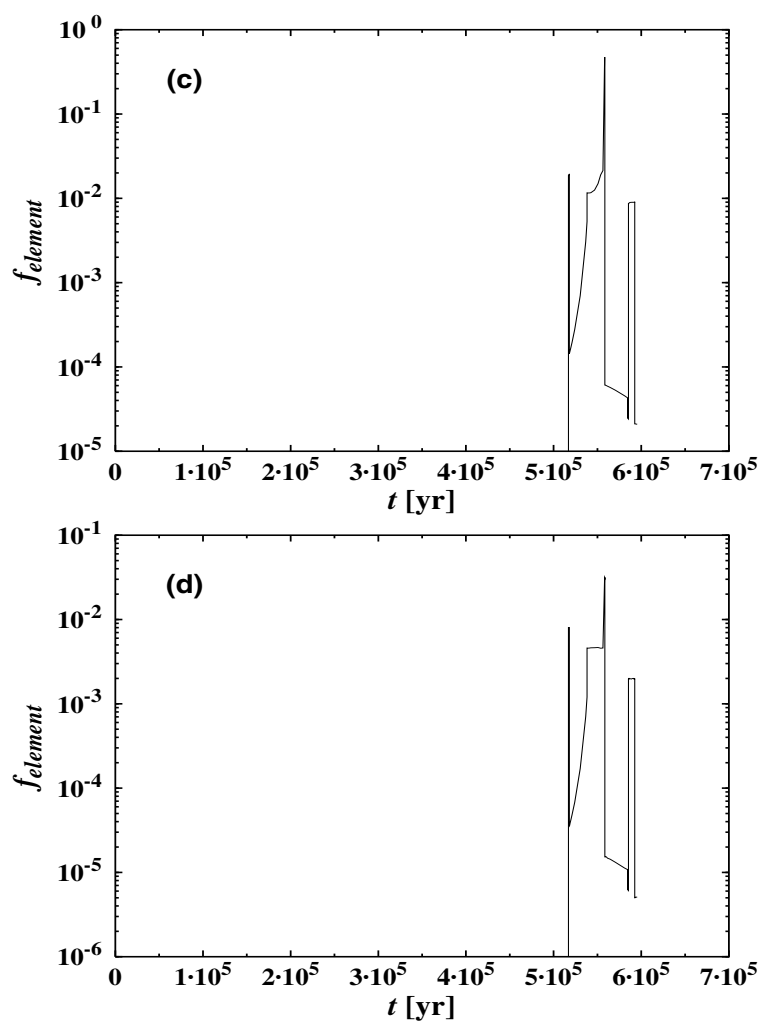

Fig. 8. As Fig. 7 c) degree of condensation of $\mathrm{Si}$ into $\mathrm{SiC}$ dust grains. d) degree of condensation of $\mathrm{C}$ into carbon grains.
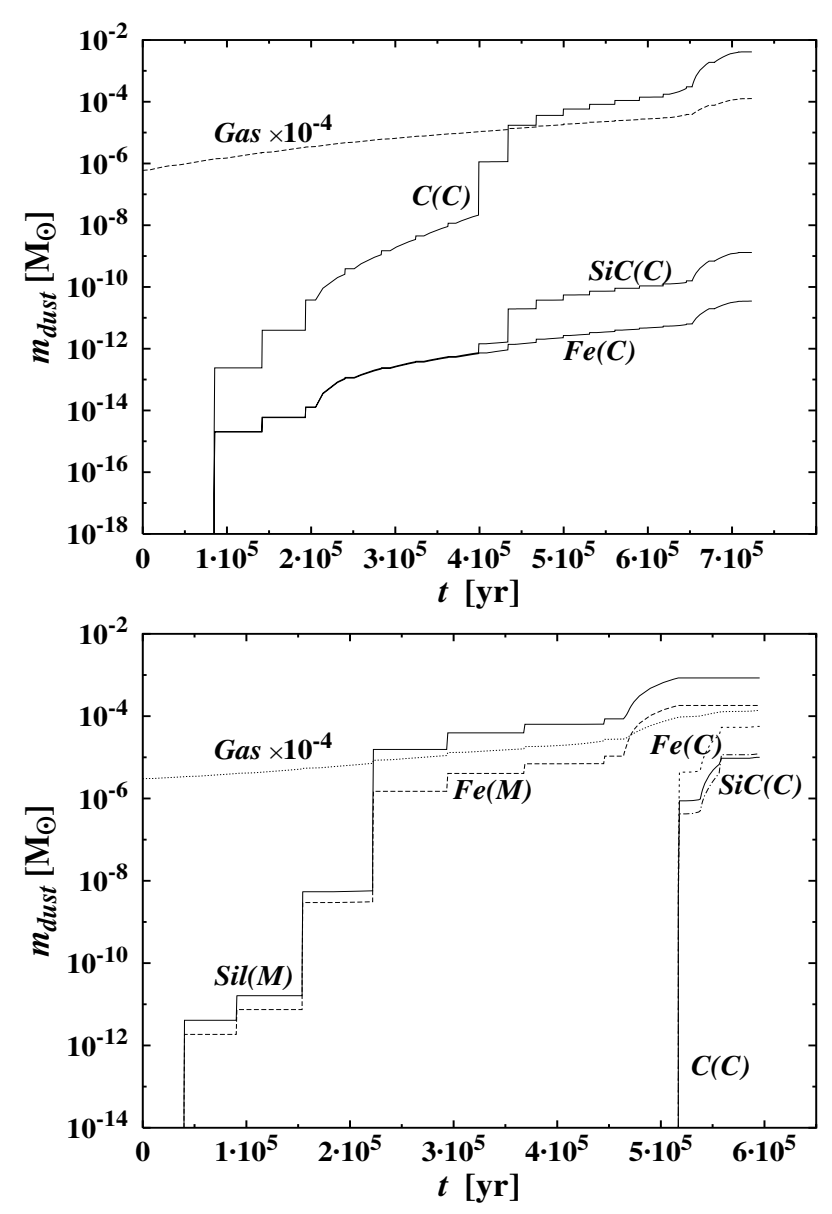

Fig. 9. Production of the different main dust species during the evolution of a star with initial mass $2 M_{\odot}$ and metallicities of $Z=0.001$ (left part) and $Z=0.02$ (right part) on the AGB. Shown are the dust masses (in solar masses) of the different dust species produced up to the instant $t$. The dust production is shown separately for the three chemically different evolutionary stages where the chemistry of the dust shell corresponds to an M-star, S-star or a C-star, which are indicated by an $\mathrm{M}, \mathrm{S}$, and $\mathrm{C}$ in brackets. The zero point of the time scale is at the first thermal pulse.

\subsection{Total dust production rates}

Dust species that may be formed during different chemical evolutionary phases (for instance silicate dust during the M-star and S-star phase) may in principle be distinguished by their trace element compositions and isotopic ratios, which may be different due to growing contributions to the stellar envelope of nuclear processed material "dredged-up" during the course of AGB-evolution. Here we do not consider such details and add up the contributions of the different chemical evolutionary phases to obtain the total output of dust mass for the different dust species. The results are presented in Table 3 for stars of different initial masses between 1 and $7 M_{\odot}$ and metallicities $Z=0.04, Z=0.02, Z=0.008, Z=0.004$, and $Z=0.001$.

The number $N_{\mathrm{p}}$ of thermal pulses for the models in Table 3 is rather high for models with initial masses $M_{*}>2 M_{\odot}$ because of the high dredge-up efficiency $\lambda$. This results in only a slow increase of the core mass, because most of the He that is freshly formed during the inter-pulse phase is mixed into the 
Table 3. Number of thermal pulses $N_{\mathrm{p}}$, total mass $M_{\text {ret }}$ returned to the ISM, duration of AGB-phase $t_{\mathrm{AGB}}$ in years, and ejected dust masses in $M_{\odot}$ for the different dust species and for the different chemistries of M-stars, S-stars, and C-stars for different initial stellar masses and different metallicities. For the $M_{*}=4 M_{\odot}$ models, two different versions are given: models without $\left(4^{-}\right)$and with $\left(4^{+}\right)$hot-bottom burning. The models with 5 and $7 M_{\odot}$ are with hot-bottom burning.

\begin{tabular}{|c|c|c|c|c|c|c|c|c|c|c|}
\hline \multirow[t]{2}{*}{$M_{*}$} & \multirow[t]{2}{*}{$N_{\mathrm{p}}$} & \multirow[t]{2}{*}{$M_{\text {ret }}$} & \multirow[t]{2}{*}{$t_{\mathrm{AGB}}$} & \multicolumn{2}{|c|}{ - silicates - } & \multicolumn{3}{|c|}{ - iron dust } & \multirow{2}{*}{$\begin{array}{c}\mathrm{SiC} \\
\mathrm{C}\end{array}$} & \multirow{2}{*}{$\begin{array}{c}\text { carbon } \\
\text { C }\end{array}$} \\
\hline & & & & M & $S$ & M & $S$ & $\mathrm{C}$ & & \\
\hline & & & & & & $Z=0.04$ & & & & \\
\hline 1.0 & 3 & 0.447 & $1.579 \times 10^{5}$ & $1.878 \times 10^{-3}$ & 0.0 & $2.677 \times 10^{-4}$ & 0.0 & 0.0 & 0.0 & 0.0 \\
\hline 1.25 & 5 & 0.688 & $2.714 \times 10^{5}$ & $3.577 \times 10^{-3}$ & 0.0 & $4.320 \times 10^{-4}$ & 0.0 & 0.0 & 0.0 & 0.0 \\
\hline 1.5 & 5 & 0.937 & $2.935 \times 10^{5}$ & $4.722 \times 10^{-3}$ & 0.0 & $4.772 \times 10^{-4}$ & 0.0 & 0.0 & 0.0 & 0.0 \\
\hline 1.7 & 6 & 1.130 & $3.601 \times 10^{5}$ & $5.617 \times 10^{-3}$ & 0.0 & $5.387 \times 10^{-4}$ & 0.0 & 0.0 & 0.0 & 0.0 \\
\hline 2.0 & 7 & 1.419 & $4.420 \times 10^{5}$ & $6.668 \times 10^{-3}$ & 0.0 & $5.467 \times 10^{-4}$ & 0.0 & 0.0 & 0.0 & 0.0 \\
\hline 2.5 & 12 & 1.902 & $8.120 \times 10^{6}$ & $1.737 \times 10^{-3}$ & $1.044 \times 10^{-7}$ & $1.006 \times 10^{-3}$ & $2.009 \times 10^{-3}$ & $1.279 \times 10^{-3}$ & $4.940 \times 10^{-4}$ & $1.087 \times 10^{-3}$ \\
\hline 3.0 & 20 & 2.388 & $1.311 \times 10^{6}$ & $1.304 \times 10^{-8}$ & $3.695 \times 10^{-11}$ & $7.131 \times 10^{-9}$ & $1.496 \times 10^{-8}$ & $1.538 \times 10^{-3}$ & $2.952 \times 10^{-3}$ & $1.175 \times 10^{-2}$ \\
\hline $4.0^{-}$ & 41 & 3.345 & $1.462 \times 10^{6}$ & $1.487 \times 10^{-8}$ & $3.293 \times 10^{-11}$ & $8.714 \times 10^{-9}$ & $1.225 \times 10^{-8}$ & $6.221 \times 10^{-5}$ & $3.640 \times 10^{-3}$ & $4.099 \times 10^{-2}$ \\
\hline $4.0^{+}$ & 39 & 3.345 & $1.381 \times 10^{6}$ & $1.136 \times 10^{-2}$ & 0.0 & $1.556 \times 10^{-3}$ & 0.0 & $3.309 \times 10^{-7}$ & $8.349 \times 10^{-5}$ & $1.141 \times 10^{-3}$ \\
\hline 5.0 & 76 & 4.257 & $1.190 \times 10^{6}$ & $1.361 \times 10^{-2}$ & 0.0 & $1.333 \times 10^{-3}$ & 0.0 & $2.116 \times 10^{-4}$ & $5.865 \times 10^{-5}$ & $9.399 \times 10^{-5}$ \\
\hline \multirow[t]{2}{*}{7.0} & 343 & 6.055 & $9.814 \times 10^{5}$ & $1.770 \times 10^{-2}$ & 0.0 & $5.563 \times 10^{-4}$ & $2.301 \times 10^{-4}$ & 0.0 & 0.0 & 0.0 \\
\hline & & & & & & $Z=0.02$ & & & & \\
\hline 1.0 & 4 & 0.408 & $2.184 \times 10^{5}$ & $2.438 \times 10^{-4}$ & 0.0 & $4.390 \times 10^{-5}$ & 0.0 & 0.0 & 0.0 & 0.0 \\
\hline 1.25 & 5 & 0.653 & $2.771 \times 10^{5}$ & $5.607 \times 10^{-4}$ & 0.0 & $7.197 \times 10^{-5}$ & 0.0 & 0.0 & 0.0 & 0.0 \\
\hline 1.5 & 6 & 0.898 & $3.458 \times 10^{5}$ & $8.093 \times 10^{-4}$ & 0.0 & $9.596 \times 10^{-5}$ & 0.0 & 0.0 & 0.0 & 0.0 \\
\hline 1.7 & 7 & 1.012 & $4.268 \times 10^{5}$ & $1.026 \times 10^{-3}$ & 0.0 & $1.601 \times 10^{-5}$ & 0.0 & 0.0 & 0.0 & 0.0 \\
\hline 2.0 & 10 & 1.383 & $5.928 \times 10^{5}$ & $8.482 \times 10^{-4}$ & 0.0 & $1.818 \times 10^{-4}$ & 0.0 & $5.374 \times 10^{-5}$ & $3.154 \times 10^{-5}$ & $3.823 \times 10^{-4}$ \\
\hline 2.5 & 16 & 1.873 & $9.579 \times 10^{5}$ & $1.143 \times 10^{-9}$ & $1.191 \times 10^{-11}$ & $5.511 \times 10^{-10}$ & $3.061 \times 10^{-9}$ & $5.350 \times 10^{-5}$ & $6.231 \times 10^{-4}$ & $5.815 \times 10^{-3}$ \\
\hline 3.0 & 25 & 2.371 & $1.471 \times 10^{6}$ & 0.0 & 0.0 & 0.0 & $3.813 \times 10^{-11}$ & $1.183 \times 10^{-6}$ & $6.945 \times 10^{-4}$ & $1.821 \times 10^{-2}$ \\
\hline $4.0^{-}$ & 52 & 3.219 & $6.010 \times 10^{5}$ & $2.986 \times 10^{-9}$ & $4.503 \times 10^{-12}$ & $1.475 \times 10^{-9}$ & $6.159 \times 10^{-10}$ & $2.799 \times 10^{-6}$ & $1.145 \times 10^{-3}$ & $1.906 \times 10^{-2}$ \\
\hline $4.0^{+}$ & 49 & 3.221 & $5.571 \times 10^{5}$ & $2.695 \times 10^{-3}$ & 0.0 & $1.787 \times 10^{-4}$ & 0.0 & $1.147 \times 10^{-8}$ & $4.109 \times 10^{-6}$ & $3.232 \times 10^{-4}$ \\
\hline 5.0 & 123 & 4.121 & $5.362 \times 10^{5}$ & $3.241 \times 10^{-3}$ & 0.0 & $1.440 \times 10^{-4}$ & $1.107 \times 10^{-4}$ & $2.151 \times 10^{-6}$ & $4.199 \times 10^{-5}$ & $1.006 \times 10^{-4}$ \\
\hline \multirow[t]{2}{*}{7.0} & 608 & 5.969 & $6.949 \times 10^{5}$ & $4.270 \times 10^{-3}$ & $2.311 \times 10^{-8}$ & $1.502 \times 10^{-4}$ & $6.075 \times 10^{-5}$ & $2.260 \times 10^{-5}$ & $4.951 \times 10^{-5}$ & $1.123 \times 10^{-4}$ \\
\hline & & & & & & $Z=0.008$ & & & & \\
\hline 1.0 & 10 & 0.357 & $7.220 \times 10^{5}$ & $1.542 \times 10^{-9}$ & 0.0 & $6.994 \times 10^{-10}$ & 0.0 & 0.0 & 0.0 & 0.0 \\
\hline 1.25 & 10 & 0.613 & $6.810 \times 10^{5}$ & $2.606 \times 10^{-6}$ & 0.0 & $2.162 \times 10^{-6}$ & 0.0 & $3.255 \times 10^{-7}$ & $1.242 \times 10^{-6}$ & $1.708 \times 10^{-4}$ \\
\hline 1.5 & 10 & 0.865 & $7.027 \times 10^{5}$ & $4.229 \times 10^{-10}$ & 0.0 & $2.351 \times 10^{-10}$ & 0.0 & $4.986 \times 10^{-8}$ & $7.115 \times 10^{-6}$ & $8.344 \times 10^{-4}$ \\
\hline 1.7 & 12 & 1.063 & $8.005 \times 10^{5}$ & $5.645 \times 10^{-11}$ & 0.0 & $2.671 \times 10^{-11}$ & 0.0 & $1.788 \times 10^{-8}$ & $9.150 \times 10^{-6}$ & $1.909 \times 10^{-3}$ \\
\hline 2.0 & 15 & 1.359 & $9.464 \times 10^{5}$ & $2.740 \times 10^{-11}$ & 0.0 & $1.581 \times 10^{-11}$ & 0.0 & $1.061 \times 10^{-8}$ & $1.298 \times 10^{-5}$ & $4.290 \times 10^{-3}$ \\
\hline 2.5 & 21 & 1.857 & $1.233 \times 10^{6}$ & $1.207 \times 10^{-12}$ & $2.060 \times 10^{-14}$ & $5.475 \times 10^{-13}$ & $6.430 \times 10^{-13}$ & $8.048 \times 10^{-9}$ & $1.809 \times 10^{-5}$ & $1.088 \times 10^{-2}$ \\
\hline 3.0 & 32 & 2.355 & $1.757 \times 10^{6}$ & 0.0 & 0.0 & 0.0 & 0.0 & $7.406 \times 10^{-9}$ & $2.128 \times 10^{-5}$ & $2.389 \times 10^{-2}$ \\
\hline $4.0^{-}$ & 89 & 3.130 & $4.581 \times 10^{5}$ & $1.162 \times 10^{-10}$ & $2.118 \times 10^{-13}$ & $5.326 \times 10^{-12}$ & $6.423 \times 10^{-12}$ & $1.776 \times 10^{-8}$ & $5.348 \times 10^{-5}$ & $1.480 \times 10^{-2}$ \\
\hline $4.0^{+}$ & 84 & 3.131 & $4.293 \times 10^{5}$ & $2.593 \times 10^{-4}$ & 0.0 & $6.776 \times 10^{-5}$ & 0.0 & $7.525 \times 10^{-7}$ & $8.954 \times 10^{-6}$ & $5.412 \times 10^{-4}$ \\
\hline 5.0 & 200 & 4.079 & $6.023 \times 10^{5}$ & $3.601 \times 10^{-4}$ & 0.0 & $8.337 \times 10^{-5}$ & 0.0 & $4.953 \times 10^{-7}$ & $9.178 \times 10^{-6}$ & $5.412 \times 10^{-4}$ \\
\hline 7.0 & 863 & 5.956 & $8.234 \times 10^{5}$ & $5.716 \times 10^{-4}$ & $2.623 \times 10^{-10}$ & $1.074 \times 10^{-4}$ & $2.112 \times 10^{-7}$ & $8.172 \times 10^{-7}$ & $1.080 \times 10^{-5}$ & $4.912 \times 10^{-4}$ \\
\hline
\end{tabular}

envelope in the next pulse. As a result, the core mass of intermediate mass stars on the thermally pulsing AGB does not grow much over the core mass at the onset of thermal pulsing. This seems to be in accord with observations (see Appendix B).

The histograms in Fig. 10 show returned dust masses for the Karakas et al. "dredge-up" parametrization. There are obvious and significant differences in the composition of the dust injected into the interstellar medium for different metallicities.
Stars with metallicity that is about solar $(Z=0.02)$ or higher predominantly form silicate dust minerals (and iron dust). Stars with Large Magellanic Cloud $(Z=0.008)$ and lower metallicity predominantly form carbon (and $\mathrm{SiC}$ ) dust.

For the $Z=0.02$ and $Z=0.04$ models, the models for low-mass stars do not become carbon stars, because of the rather low dredge up efficiency predicted by the Karakas et al. "dredge-up" parametrization for such stars. The tabulation in 
Table 3. continued.

\begin{tabular}{|c|c|c|c|c|c|c|c|c|c|c|}
\hline \multirow[t]{2}{*}{$\overline{M_{*}}$} & \multirow[t]{2}{*}{$\overline{N_{\mathrm{p}}}$} & \multirow[t]{2}{*}{$\overline{M_{\text {ret }}}$} & \multirow[t]{2}{*}{$t_{\mathrm{AGB}}$} & \multicolumn{2}{|c|}{ - silicates - } & \multicolumn{3}{|c|}{ - iron dust - } & \multirow{2}{*}{$\begin{array}{c}\mathrm{SiC} \\
\mathrm{C}\end{array}$} & \multirow{2}{*}{$\begin{array}{c}\text { carbon } \\
\text { C }\end{array}$} \\
\hline & & & & M & S & M & S & $\mathrm{C}$ & & \\
\hline \multicolumn{11}{|c|}{$Z=0.004$} \\
\hline 1.0 & 12 & 0.335 & $9.927 \times 10^{5}$ & $1.761^{-10}$ & 0.0 & $7.857 \times 10^{-11}$ & 0.0 & 0.0 & 0.0 & 0.0 \\
\hline 1.25 & 12 & 0.598 & $8.917 \times 10^{5}$ & $6.030^{-13}$ & 0.0 & $2.855 \times 10^{-13}$ & 0.0 & $4.083 \times 10^{-10}$ & $7.135 \times 10^{-8}$ & $5.415 \times 10^{-4}$ \\
\hline 1.5 & 12 & 0.853 & $8.681 \times 10^{5}$ & $1.342^{-13}$ & 0.0 & $6.077 \times 10^{-14}$ & 0.0 & $6.005 \times 10^{-10}$ & $1.600 \times 10^{-7}$ & $1.599 \times 10^{-3}$ \\
\hline 1.7 & 14 & 1.052 & $9.564 \times 10^{5}$ & $1.121^{-13}$ & 0.0 & $5.094 \times 10^{-14}$ & 0.0 & $6.225 \times 10^{-10}$ & $1.991 \times 10^{-7}$ & $3.001 \times 10^{-3}$ \\
\hline 2.0 & 17 & 1.348 & $1.068 \times 10^{6}$ & $9.612^{-14}$ & 0.0 & $4.385 \times 10^{-14}$ & 0.0 & $7.546 \times 10^{-10}$ & $2.829 \times 10^{-7}$ & $5.570 \times 10^{-3}$ \\
\hline 2.5 & 23 & 1.848 & $1.366 \times 10^{6}$ & $8.231^{-14}$ & 0.0 & $3.785 \times 10^{-14}$ & 0.0 & $8.649 \times 10^{-10}$ & $3.796 \times 10^{-7}$ & $1.293 \times 10^{-2}$ \\
\hline 3.0 & 35 & 2.355 & $1.844 \times 10^{6}$ & 0.0 & 0.0 & 0.0 & 0.0 & $9.471 \times 10^{-10}$ & $5.016 \times 10^{-7}$ & $2.655 \times 10^{-2}$ \\
\hline $4.0^{-}$ & 115 & 3.134 & $6.191 \times 10^{5}$ & $9.193^{-12}$ & 0.0 & $4.199 \times 10^{-12}$ & 0.0 & $1.710 \times 10^{-9}$ & $1.509 \times 10^{-6}$ & $2.024 \times 10^{-2}$ \\
\hline $4.0^{+}$ & 108 & 3.134 & $5.761 \times 10^{5}$ & $1.886^{-7}$ & 0.0 & $1.016 \times 10^{-7}$ & 0.0 & $1.309 \times 10^{-8}$ & $3.393 \times 10^{-7}$ & $7.802 \times 10^{-4}$ \\
\hline 5.0 & 248 & 4.074 & $7.333 \times 10^{5}$ & $4.145^{-7}$ & $3.363 \times 10^{-11}$ & $2.209 \times 10^{-7}$ & $5.507 \times 10^{-9}$ & $1.094 \times 10^{-8}$ & $3.435 \times 10^{-7}$ & $6.840 \times 10^{-4}$ \\
\hline 7.0 & 1113 & 5.938 & $9.538 \times 10^{5}$ & $1.148^{-6}$ & 0.0 & $5.513 \times 10^{-7}$ & 0.0 & $2.002 \times 10^{-8}$ & $5.180 \times 10^{-7}$ & $6.728 \times 10^{-4}$ \\
\hline \multicolumn{11}{|c|}{$Z=0.001$} \\
\hline 1.0 & 18 & 0.301 & $1.879 \times 10^{6}$ & $9.844 \times 10^{-14}$ & 0.0 & $4.455 \times 10^{-14}$ & 0.0 & 0.0 & 0.0 & 0.0 \\
\hline 1.25 & 14 & 0.575 & $1.173 \times 10^{6}$ & 0.0 & 0.0 & 0.0 & 0.0 & $9.581 \times 10^{-12}$ & $1.353 \times 10^{-10}$ & $3.031 \times 10^{-4}$ \\
\hline 1.5 & 14 & 0.822 & $8.924 \times 10^{5}$ & 0.0 & 0.0 & 0.0 & 0.0 & $2.251 \times 10^{-11}$ & $7.036 \times 10^{-10}$ & $1.899 \times 10^{-3}$ \\
\hline 1.7 & 16 & 1.010 & $8.065 \times 10^{5}$ & 0.0 & 0.0 & 0.0 & 0.0 & $2.729 \times 10^{-11}$ & $9.983 \times 10^{-10}$ & $3.152 \times 10^{-3}$ \\
\hline 2.0 & 20 & 1.282 & $7.103 \times 10^{5}$ & $2.375^{-15}$ & 0.0 & $1.091 \times 10^{-15}$ & 0.0 & $3.483 \times 10^{-11}$ & $1.359 \times 10^{-9}$ & $4.580 \times 10^{-3}$ \\
\hline 2.5 & 29 & 1.750 & $6.281 \times 10^{5}$ & $5.152^{-15}$ & 0.0 & $2.376 \times 10^{-15}$ & 0.0 & $4.893 \times 10^{-11}$ & $2.090 \times 10^{-9}$ & $8.079 \times 10^{-3}$ \\
\hline 3.0 & 49 & 2.200 & $5.804 \times 10^{5}$ & $7.270^{-14}$ & 0.0 & $3.420 \times 10^{-14}$ & 0.0 & $6.255 \times 10^{-11}$ & $3.016 \times 10^{-9}$ & $1.192 \times 10^{-2}$ \\
\hline $4.0^{-}$ & 133 & 3.134 & $7.381 \times 10^{5}$ & $1.768^{-13}$ & 0.0 & $8.143 \times 10^{-14}$ & 0.0 & $7.993 \times 10^{-11}$ & $4.627 \times 10^{-9}$ & $2.436 \times 10^{-2}$ \\
\hline $4.0^{+}$ & 123 & 3.133 & $6.825 \times 10^{5}$ & $2.191^{-9}$ & 0.0 & $1.048 \times 10^{-9}$ & 0.0 & $2.304 \times 10^{-10}$ & $1.617 \times 10^{-9}$ & $9.213 \times 10^{-4}$ \\
\hline 5.0 & 284 & 4.073 & $8.493 \times 10^{5}$ & $3.674^{-9}$ & 0.0 & $1.739 \times 10^{-9}$ & 0.0 & $3.108 \times 10^{-10}$ & $1.719 \times 10^{-9}$ & $9.034 \times 10^{-4}$ \\
\hline 7.0 & 1352 & 5.924 & $1.031 \times 10^{6}$ & $7.030^{-9}$ & 0.0 & $3.318 \times 10^{-9}$ & 0.0 & $3.379 \times 10^{-10}$ & $2.102 \times 10^{-9}$ & $8.850 \times 10^{-4}$ \\
\hline
\end{tabular}

Groenewegen et al. (1995) shows that for Pop I, only stars with $M_{*} \gtrsim 1.5, \ldots, 1.8 M_{\odot}$ seem to become carbon stars. For our models with $Z=0.02$, the $M_{*}=2 M_{\odot}$ model is the lowest mass model to become a $\mathrm{C}$ star, roughly in accord with the observational findings. For $Z=0.04$, the $M_{*}=2.5 M_{\odot}$ model is the lowest mass model to become a carbon star, because the higher initial excess abundance of $\mathrm{O}$ requires more $\mathrm{C}$ to be "dredged-up" to convert the star into a carbon star.

In the mass region from the lower limit for becoming a carbon star up to $4 M_{\odot}$, the Pop I stars in our models mainly produce carbon dust (and $\mathrm{SiC}$ ). During most of their evolution as oxygen rich objects, they are semiregular or irregular variables or Miras with rather short periods and low mass-loss rates. This evolutionary phase does not contribute significantly to the total dust production, though there are brief phases following a thermal pulse with rather high mass-loss rates $\left(\dot{M}>10^{-5} M_{\odot} \mathrm{yr}^{-1}\right)$. Only till the end of their AGB evolution, and if the total mass is significantly reduced and the effective temperature becomes low, the pulsation period becomes long enough (cf. Eq. (62)) that massive mass loss also commence during the inter-pulse phases. But then their envelopes are already carbon-rich.

For models with initial mass $M_{*}>4 M_{\odot}$, we have assumed that hot bottom burning occurs. For $Z=0.008, Z=0.02$, and $Z=0.04$, such stars mainly produce silicate dust because the "dredged-up" carbon is converted into ${ }^{14} \mathrm{~N}$. Only at the end of their evolution, if the envelope mass is too small for hot bottom burning to operate, they become $\mathrm{C}$ stars and produce some carbon dust.

Generally the dust production increases with increasing stellar mass, but the dust output decreases with increasing initial mass for the models with the highest masses, which are also that which experience hot bottom burning, because these stars are too hot and too massive for efficient dust production for most of their lives as thermally pulsing AGB stars. Due to our presently strongly simplified treatment of this process, the results for the dust production of stars experiencing hot bottom burning are only approximate.

For applications in model calculations of the evolution of interstellar dust, one can interpolate linearly in $M_{*}$ and $\log Z$ in the table for the dust-mass return of the different species. Numbers given for the dust mass below $10^{-10} M_{\odot}$ are probably unrealistic, since our method for calculating dust growth then becomes dependent on the assumptions with respect to the nature and number density of the seed particles, which are rather crude. For numerical purposes, it is better to replace tabular values below $10^{-10}$ by zero.

\subsection{Mass fraction of returned dust}

One conspicuous feature of the calculated dust production rates is that only a small fraction of the abundant refractory elements returned to the ISM is really condensed into dust grains. The 

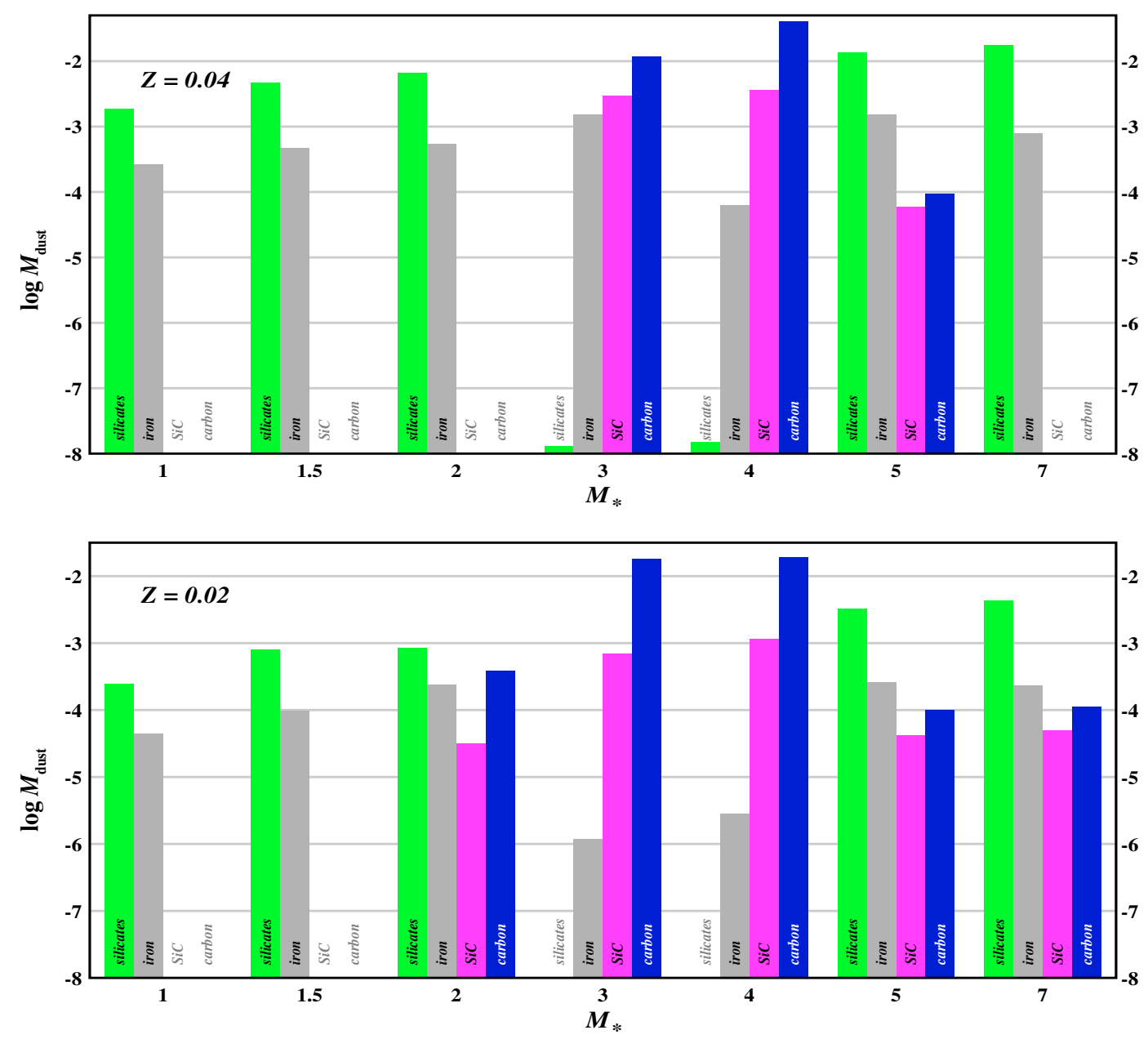

Fig. 10. Returned dust mass in solar masses for the different dust species by stars of different initial masses $M_{*}$ and metallicities.

mass fraction of the total dust mass returned is much lower than $Z$ in all cases, which essentially corresponds to the mass fraction of condensible elements. Most of the returned mass of the condensible elements remains in the gas phase, since the stars spend most of their evolution time on the AGB in phases with low mass-loss rates, where only small amounts of dust are produced. The stars then appear observationally as reddened but optically visible objects. This dominance of low mass-loss phases results from the prescriptions for massloss used in the present model calculation, that discriminate between massloss (i) by semiregular and irregular variables and Miras with short periods; (ii) by Miras with long periods; and (iii) by dust-enshrouded objects. This discrimination is not made in other synthetic AGB model calculations, but it is necessary if one is interested in calculating dust formation, since observations clearly show that real AGB stars have very different mass-loss properties and dust-formation efficiencies in these different evolutionary stages.

As in other model calculations, the stars in the present calculation lose a considerable part of their envelope mass during a superwind phase that is usually associated with the dust-enshrouded objects where the stars are efficient dust factories. However, prior to this, they already lose much of their envelope mass as SRas, SRb, Lbs, and Miras with low pulsational periods, where mass-loss rates are lower than in the final superwind phase, but not negligibly small. During these periods the mass-loss rates are too low to produce much dust, but the total amount of mass lost is significant. This can clearly be seen from Fig. 9, which shows the cumulated dust and gas mass return for a $2 M_{\odot}$ star as an example. One sees that nearly all of the dust is produced in the final phase, which also contributes a major part of the total gas loss, but not in such a pronounced way as for the dust.

Since in the ISM the refractory elements are found to be more or less completely depleted from the gas phase, the notcondensed fraction of refractory elements from the stellar wind condenses onto dust grains later in the ISM.

The relation between the core mass at the end of AGB evolution to the initial main sequence mass of the star is a test of the reliability of the calculated models. This is considered in Appendix B.

\section{Concluding remarks}

In this paper we have attempted to calculate the composition of the dust mixture and amounts of dust returned by AGB stars 

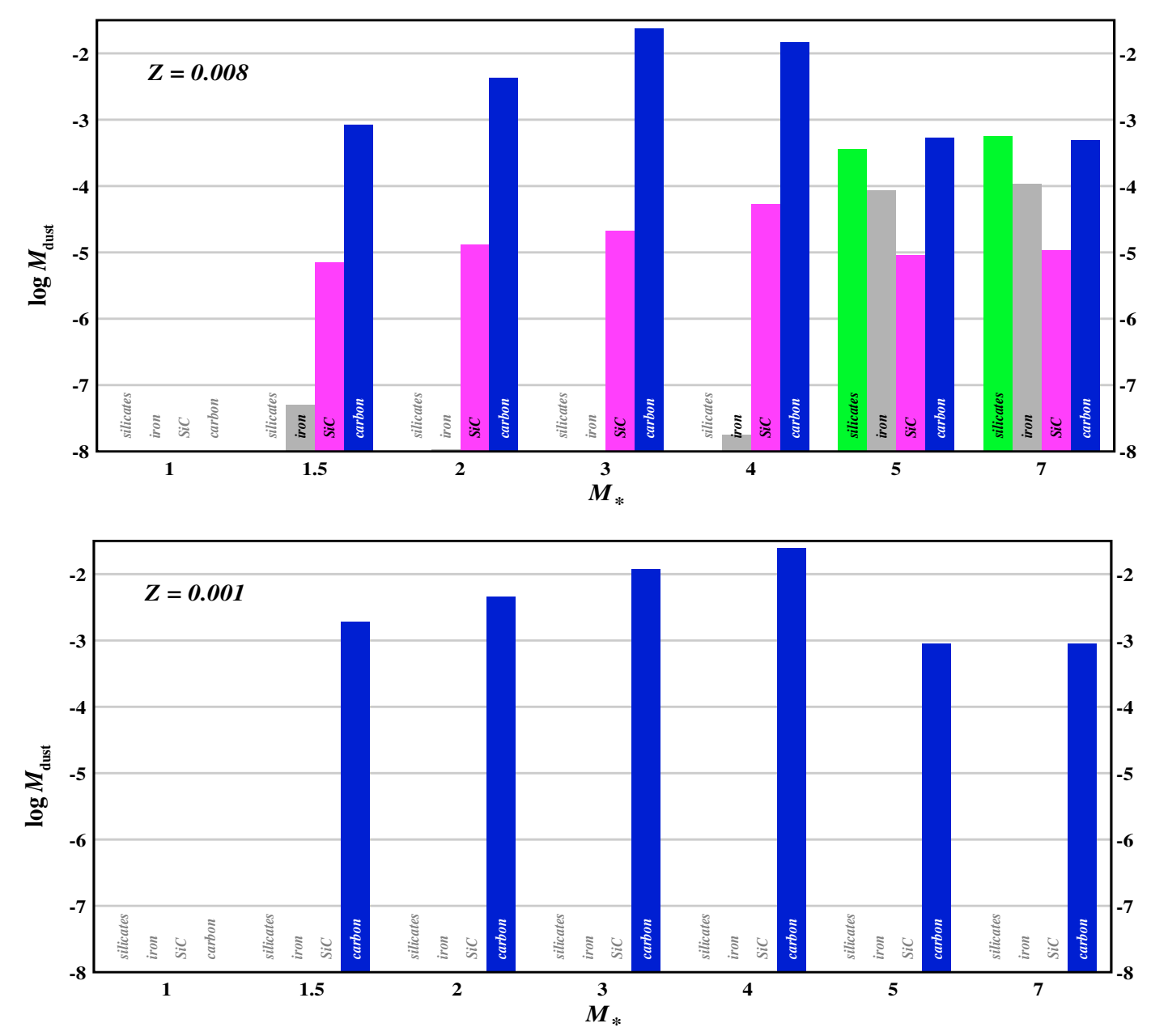

Fig. 10. continued.

into the interstellar medium. The model calculations are based on a combination of models

- for the outflows from AGB stars, which also determine the composition and quantities of dust formed in the outflow for the varying $\mathrm{C} / \mathrm{O}$ abundance ratio during AGB evolution; and

- for synthetic AGB evolution.

Both types of models contain considerable simplifications, without which such a calculation would presently hardly be possible. The results can for this reason, only be considered as preliminary, but we expect that they already give the right orders of magnitude and can be used as input for model calculations of the evolution of the dust component of the interstellar medium.

Probably the severest restriction comes from the rather schematic treatment of the luminosity variations after a thermal pulse in the approximation originally developed by Groenewegen \& de Jong (1993), which we used in order to reduce the number of wind models to be calculated. Since in the final version of the program, calculation of wind models is computationaly less expensive than in its first versions, the more accurate parameterizations of Wagenhuber \& Groenewegen (1998) will be used in future calculations. Some other simplifications, like the neglect of grain drift, will also be relaxed.

The second restriction on the accuracy of the present models comes from the also rather crude treatment of hot bottom burning. An improvement is in preparation by using envelope integrations like in Marigo et al. (1998).

A further essential ingredient of our model calculation is the prescription of mass-loss rates, which differs considerably from other calculations of synthetic AGB evolution. This modification is dictated by requirements of the dust condensation calculations, which depend much more critically on the massloss rates than does the stellar evolution itself, for which a more overall treatment seems to suffice.

The purpose of our calculations of dust injection rates into the interstellar medium is to prepare the necessary input data for modeling the evolution of the dust component in the interstellar medium of galaxies in models like the model developed by Dwek (1998). Results for this will be presented in a separate paper.

Acknowledgements. This work is part of a project of the special research project SFB 439 "Galaxies in the Young Universe", which is supported by the Deutsche Forschungsgemeinschaft (DFG). 


\section{References}

Alexander, C. M. O'D. 1997, in Astrophysical Implications of the Laboratory Study of Presolar Materials, ed. T. J. Bernatowicz, \& E. Zinner (New York: AIP), AIP Conf. Proc., 402, 567

Allende Prieto, C., Lambert, D. L., \& Asplund, M. 2001, ApJ, 556, L63

Allende Prieto, C., Lambert, D. L., \& Asplund, M. 2002, ApJ, 573, L137

Anders, E., \& Grevesse, N. 1989, Geochim. Cosmochim. Acta, 53, 197

Arndt, T. U., Fleischer, A. J., \& Sedlmayr, E. 1997, A\&A, 327, 614

Bagnulo, S., Doyle, J. G., \& Andretta, V. 1998, MNRAS, 296, 545

Bergeat, J., Knapik, A., \& Rutily, B. 2002, A\&A, 390, 967

Bernatowicz, T. J., Cowsik, R., Gibbons, P. C., et al. 1996, ApJ, 472, 760

Bernatowicz, T. J., \& Cowsik, R. 1997, in Astrophysical Implications of the Laboratory Study of Presolar Materials, ed. T. J. Bernatowicz, \& E. Zinner (New York: AIP), AIP Conf. Proc., 402, 451

Blöcker, T. 1995, A\&A, 297, 727

Blöcker, T. 2001, Ap\&SS, 275, 1

Bowen, G. H. 1988, ApJ, 329, 299

Charbonnel, C., Meynet, G., Maeder, A., Schaller, G., \& Schaerer, D. 1993, A\&AS, 101, 415

Cherchneff, I. 1998, in The Molecular Astrophysics of Stars and Galaxies, ed. T. W. Hartquist, \& D. A. Williams (Oxford: Clarendon Press), 265

Cherchneff, I., Barker, J. R., \& Tielens, A. G. G. M. 1992, ApJ, 401, 269

Chen, P. S., \& Kwok, S. 1993, ApJ, 416, 769

Claver, C. F., Liebert, J., Bergeron, P., \& Koester, D. 2001, ApJ, 563, 987

Clayton, D. D. 1968, Principles of stellar evolution and Nucleosynthesis (New York: McGraw-Hill)

Dominik, C., Gail, H.-P., Sedlmayr, E., \& Winters, M. 1990, A\&A, 240,365

Dupree, A. K. 1986, ARA\&A, 24, 377

Dupree, A. K., \& Reimers, D. 1987, in Exploring the universe in the ultraviolet with the IUE satellite, ed. Y. Kondo et al. (Dordrecht: Reidel), 321

Dwek, E. 1998, ApJ, 501, 643

Ferrarotti, A. S., \& Gail, H.-P. 2001, A\&A, 371, 133

Ferrarotti, A. S., \& Gail, H.-P. 2002, A\&A, 382, 256

Ferrarotti, A. S., \& Gail, H.-P. 2003, A\&A, 398, 1029

Feuchtinger, M. U., Dorfi, E. A., \& Höfner, S. 1993, A\&A, 273, 513

Finley, D. S., Koester, D., \& Basri, G. 1997, ApJ, 488, 375

Fleischer, A. J., Gauger, A., \& Sedlmayr, E. 1991, A\&A, 242, L1

Fleischer, A. J., Gauger, A., \& Sedlmayr, E. 1992, A\&A, 266, 321

Gail, H.-P. 2003, in Astromineralogy, Lecture Notes in Physics 609, ed. Th. Henning (Heidelberg: Springer), 55

Gail, H.-P., \& Sedlmayr, E. 1985, A\&A, 148, 183

Gail, H.-P., \& Sedlmayr, E. 1987, A\&A, 171, 197

Gail, H.-P., \& Sedlmayr, E. 1988, A\&A, 206, 153

Gail, H.-P., \& Sedlmayr, E. 1998a, in The Molecular Astrophysics of Stars and Galaxies, ed. T. W. Hartquist, \& D. A. Wiliams (Oxford: Clarendon Press), 285

Gail, H.-P., \& Sedlmayr, E. 1998b, Faraday Discussion, 109, 303

Gail, H.-P., \& Sedlmayr, E. 1999, A\&A, 347, 594

Gautschy, A. 1999, A\&A, 349, 209

Gehrz, R. D. 1989, in Interstellar Dust, ed. L. J. Allamandola, \& A. G. G. M. Tielens (Dordrecht: Kluwer), IAU Symp., 135, 445
Girardi, L., Bressan, A., Bertelli, G., \& Chiosi, C. 2000, A\&AS, 141, 371

Goeres, A. 1993, Rev. Mod. Astron., 6, 165

Golub, G. H., \& Ortega, J. M. 1992, Scientific Computing and Differential Equations (Boston: Academic Press)

González Delgado, D., Olofsson, H., Kerschbaum, F., et al. 2003, A\&A, 411, 123

Grevesse, N., \& Noels, A. 1993, in Origin and Evolution of the Elements, ed. N. Prantzos, E. Vangioni-Flam, \& M. Cassé (Cambridge: Cambridge University Press), 14

Grevesse, N., \& Sauval, A. J. 1998, Space Sci. Rev., 85, 161

Groenewegen, M. A. T. 1995, A\&A, 293, 463

Groenewegen, M. A. T., \& de Jong, T. 1993, A\&A, 267, 410

Groenewegen, M. A. T., \& de Jong, T. 1994a, A\&A, 282, 127

Groenewegen, M. A. T., \& de Jong, T. 1994b, A\&A, 283, 463

Groenewegen, M. A. T., \& de Jong, T. 1994c, A\&A, 288, 782

Groenewegen, M. A. T., van den Hoek, L. B., \& de Jong, T. 1995, A\&A, 293, 381

Groenewegen, M. A. T., Whitelock, P. A., Smith, C. H., \& Kerschbaum, F. 1998, MNRAS, 293, 18

Harper, G. 1996, in Cool Stars, Stellar Systems, and the Sun, ed. R. Pallavicini, \& A. K. Dupree, ASP Conf. Ser., 109, 481

Herwig, F. 2001, Ap\&SS, 275, 15

Höfner, S., \& Dorfi, E. A. 1997, A\&A, 319, 648

Höfner, S., Jørgensen, U. G., Loidl, R., \& Aringer, B. 1998, A\&A, 340,497

van den Hoek, L. B., \& Groenewegen, M. A. T. 1997, A\&AS, 123, 305

Hughes, S. M. G., \& Wood, P. R. 1990, AJ, 99, 784

Hurley, J. J., Pols, O. R., \& Tout, C. A. 2000, MNRAS, 315, 543

Iben, I. 1985, QJRAS, 26, 1

Jaschek, C., \& Jaschek, M. 1987, The classification of stars (Cambrige: Cambridge University Press)

Jeong, K. S., Winters, J. M., Le Bertre, T., \& Sedlmayr, E. 2003, A\&A, 407, 191

Jones, A. P., Tielens, A. G. G. M., Hollenbach, D. J., \& McKee, C. F. 1997, in Astrophysical Implications of the Laboratory Study of Presolar Materials, ed. T. J. Bernatowicz, \& E. Zinner (New York: AIP), AIP Conf. Proc., 402, 595

Jones, A. P. 2000, J. Geophys. Res., 105, 10257

Karakas, A. I., Latanzio, J. C., \& Pols, O. 2002, PASA, 19, 515

Kerschbaum, F. 1999, A\&A, 351, 627

Kerschbaum, F., \& Olofsson, H. 1998, A\&A, 336, 654

Kerschbaum, F., \& Olofsson, H. 1999, A\&AS, 138, 299

Kerschbaum, F., Olofsson, H., \& Hron, J. 1996, A\&A, 311, 273

Kudritzki, R., \& Reimers, D. 1978, A\&A, 70, 227

Lamers, H. J. G. L. M., \& Cassinelli, J. P. 1999, Introduction to stellar winds (Cambridge: Cambridge University Press)

Lebzelter, T., \& Hron, J. 2003, A\&A, 411, 533

Lloyd Evans, T., \& Little-Marenin, I. R. 1999, MNRAS, 304, 421

Lodders, K., \& Fegley, B. 1995, Meteoritics, 30, 661

Lucy, L. B. 1971, ApJ, 163, 95

Lucy, L. B. 1976, ApJ, 205, 482

Marigo, P. 1998, A\&A, 340, 463

Marigo, P. 2001, A\&A, 370, 194

Marigo, P. 2002, A\&A, 387, 507

Marigo, P., Bressan, A., \& Chiosi, C. 1996, A\&A, 313, 545

Marigo, P., Bressan, A., \& Chiosi, C. 1998, A\&A, 331, 564

Marigo, P., Girardi, L., \& Bressan, A. 1999, A\&A, 344, 123

Marigo, P., Bernard-Salas, J., Pottasch, S. R., Tielens, A. G. G. M., \& Wesselius, P. R. 2003, A\&A, 409, 619

Messenger, S., Keller, L. P., Stadermann, F. J., Walker, R. M., \& Zinner, E. 2003, Science, 300, 105 
Mouhcine, M., \& Lançon, A. 2002, A\&A, 393, 149

Molster, F. J., Waters, L. B. F. M., Tielens, A. G. G. M., \& Barlow, M. J. 2002a, A\&A, 382, 184

Molster, F. J., Waters, L. B. F. M., \& Tielens, A. G. G. M. 2002b, A\&A, 382, 222

Molster, F. J., Waters, L. B. F. M., Tielens, A. G. G. M., Koike, C., \& Chihara, H. 2002c, A\&A, 382, 241

Mowlavi, N. 1999, A\&A, 344, 617

Olofsson, H., González Delgado, D., Kerschbaum, F., \& Schöier, F. L. 2002, A\&A, 391, 1053

Ossenkopf, V., Henning, Th., \& Mathis, J. S. 1992, A\&A, 261, 567

Piccirillo, J. 1980, MNRAS, 190, 441

Reddy, B. E., Tomkin, J., Lambert, D. L., \& Allende Prieto, C. 2003, MNRAS, 340, 304

Reimers, D. 1975, in Problems in Stellar Atmospheres and Envelopes, ed. B. Baschek et al. (Berlin: Springer), 229

Renzini, A., \& Voli, M. 1981, A\&A, 94, 175

Schaller, G., Schaerer, D., Meynet, G., \& Maeder, A. 1992, A\&AS, 96, 269

Schaerer, D., Meynet, G., Maeder, A., \& Schaller, G. 1993, A\&AS, 98, 523

Schröder, K.-P., \& Sedlmayr, E. 2001, A\&A, 366, 913

Schröder, K.-P., Winters, J.-M., \& Sedlmayr, E. 1999, A\&A, 349, 898

Schöier, F. L., \& Olofsson, H. 2001, A\&A, 368, 969

Smith, V. V., \& Lambert, D. L. 1990, ApJS, 72, 387

Straniero, O., Chieffi, A., \& Limongi, M., et al. 1997, ApJ, 478, 332

Thielemann, F.-K., Argast, D., \& Brachwitz, F., et al. 2002 [arXiv: astro-ph/0202453]
Tielens, A. G. G. M. 1997, in Formation and Evolution of Solids in Space, ed. J. M. Greenberg, \& A. Li (Dordrecht: Kluwer), 331

Tielens, A. G. G. M. 1998, ApJ, 499, 267

Tout, C. A., Aarseth, S. J., Pols, O. R., \& Eggleton, P. P. 1997, MNRAS, 291, 732

Tuchman, Y., Sack, N., \& Barkat, Z. 1979, ApJ, 234, 217

Vassiliadis, E., \& Wood, P. R. 1993, ApJ, 413, 641

Wachter, A., Schröder, K.-P., Winters, J. M., Arndt, T. U., \& Sedlmayr, E. 2002, A\&A, 384, 452

Wagenhuber, J., \& Groenewegen, M. A. T. 1998, A\&A, 340, 183

Weidemann, V. 1990, ARA\&A, 28, 103

Weidemann, V. 2000, A\&A, 363, 647

Willson, L. A. 2000, ARA\&A, 38, 573

Winters, J. M., Fleischer, A. J., Le Bertre, T., \& Sedlmayr, E. 1997, A\&A, 326, 305

Winters, J. M., Le Bertre, T., Jeong, K. S., Helling, C., \& Sedlmayr, E. 2000, A\&A, 361, 641

Winters, J. M., Le Bertre, T., Jeong, K. S., Nyman, L.-Å., \& Epchtein, N. 2003, A\&A, 409, 715

Wood, P. R. 1990, in From Miras to Planetary Nebulae, ed. M. O. Mennessier, \& A. Omont (Editions Frontières), 67

Wood, P. R., \& Zarro, D. M. 1981, ApJ, 247, 247

Wood, P. R., Alcock, C., Allsman, R. A., et al. 1999, in Asymptotic Giant Branch Stars, ed. T. Le Bertre, A. Lèbre, \& C. Waelkens, IAU Symp., 191, 151

Xiong, D. R., Deng, L., \& Cheng, Q. L. 1998, ApJ, 499, 355 
A. S. Ferrarotti and H.-P. Gail: Dust production by AGB stars, Online Material $p 1$

\section{Online Material}




\section{Appendix A: Dust formation: some examples}

We have calculated a grid of wind models for varying $\mathrm{C} / \mathrm{O}$ abundance ratios and mass-loss rates in order to demonstrate the variation of the condensation of the most abundant dust forming elements into different dust species on the AGB. For these calculations we assume fixed values for the stellar parameters: a luminosity of $L_{*}=10^{4} L_{\odot}$, a stellar mass of $M_{*}=1 M_{\odot}$ and the following effective temperatures: $T_{\mathrm{eff}}=$ $3000 \mathrm{~K}$ for M-stars and $T_{\text {eff }}=2500 \mathrm{~K}$ for S- and C-stars (e.g. Kerschbaum 1999; Bergeat et al. 2002).

Since for abundance ratios $\mathrm{C} / \mathrm{O}$ between about 0.9 and 1.0 no silicate or carbon dust can be formed, the radiation pressure on dust is inefficient for the chosen value of $L_{*}$ and in many cases there exists no dust driven wind. In this case the wind is driven by some other mechanism, for instance pulsations. We proceed therefore in the following way: for a given set of parameters $\dot{M}$ and $\mathrm{C} / \mathrm{O}$ abundance ratios we first try to calculate a dust driven wind model. If it turns out that radiation pressure on dust is insufficient to drive the wind we calculate instead a model with a constant outflow velocity of $5 \mathrm{~km} \mathrm{~s}^{-1}$. The velocity is assumed to be constant in the dust forming zone for the reason that the main wind acceleration for a wind driven by a mechanism different from radiation pressure on grains has to occur in a region far inside of the dust formation region. The wind in this case should have already nearly approached its terminal velocity (cf. Harper 1996). A velocity of $5 \mathrm{~km} \mathrm{~s}^{-1}$ is chosen because outflow velocities of AGB stars with low dust production observationally are known to have such low outflow velocities, see Sect. 4.

\section{A.1. Element abundances}

For calculating the dust formation we have to specify the abundances of the main dust forming elements, i.e. $\mathrm{C}, \mathrm{O}, \mathrm{Mg}, \mathrm{Si}, \mathrm{S}$, and $\mathrm{Fe}$. The abundances of $\mathrm{C}$ and $\mathrm{O}$ change during the evolution of AGB stars due to the "dredge-up" processes. The abundances of the other elements are fixed by the initial composition of the star since burning temperatures in low and intermediate mass stars are not high enough for significant abundance changes of $\mathrm{Mg}, \mathrm{Si}, \mathrm{S}$ or Fe to occur. In our synthetic AGB evolution models the abundance changes of $\mathrm{C}$ and $\mathrm{O}$ by "dredge-up" are calculated as part of the model calculation. At this place we assume the $\mathrm{C} / \mathrm{O}$ abundance ratio to be a free parameter, which is varied between reasonable limits. For the calculations presented in this section we use the solar abundances of Grevesse \& Sauval (1998) with the correction for $\mathrm{O}$ by Allende Prieto et al. (2001) and for C by Allende Prieto et al. (2002), see Table 1. For comparison, the Table also shows the most recent set of abundances determined by Reddy et al. 2003 for a sample of 181 galactic $F$ and $G$ dwarfs from the thin galactic disk, which we assume to be more representative for the cosmic element abundance of Pop I stars.

If the $\mathrm{C} / \mathrm{O}$ abundance ratio is increased, the abundance of available oxygen to form silicate minerals decreases. While olivine-type dust requires four and pyroxene-type dust three $\mathrm{O}$ atoms per $\mathrm{Si}$ atom, formation of quartz-type dust only requires two $\mathrm{O}$ atoms for each $\mathrm{Si}$ atom. One expects a strong change of the mineral composition to occur if even insufficient oxygen is available to form $\mathrm{SiO}_{2}$, which happens to occur above a $\mathrm{C}$ abundance of

$\epsilon_{\mathrm{C}, \mathrm{qu}}=\epsilon_{\mathrm{O}}-2 \epsilon_{\mathrm{Si}}$,

i.e. for the abundances of Table 1 at $\mathrm{C} / \mathrm{O}=0.85$.

The chemistry changes to the typical carbon dominated chemistry, if also insufficient $\mathrm{O}$ is available for the formation of gas phase $\mathrm{SiO}$ molecules, which occurs above a $\mathrm{C}$ abundance of

$\epsilon_{\mathrm{C}, \mathrm{carb}}=\epsilon_{\mathrm{O}}-\epsilon_{\mathrm{Si}}+\epsilon_{\mathrm{S}}$,

since for an oxygen rich mixture the $\mathrm{S}$ in the gas phase is completely bound in $\mathrm{SiS}$ and the excess of $\mathrm{Si}$ is bound in $\mathrm{SiO}$. For the abundances of Table 1 this limit is at $\mathrm{C} / \mathrm{O}=0.97$. For simplicity we replace the last limit by $\mathrm{C} / \mathrm{O}=1.00$.

The transition from the silicate dominated mineral composition of $\mathrm{M}$ stars to the peculiar mixture of solids of $\mathrm{S}$ stars thus can be expected to occur around $\mathrm{C} / \mathrm{O}=0.85$ while the carbon dominated dust mixture starts above $\mathrm{C} / \mathrm{O} \approx 1.00$. For $\mathrm{C} / \mathrm{O}$ abundance ratios in between one has the iron dominated dust mixture of S stars. These transitions can be seen in more detail in the numerical results presented below.

This definition of S stars refers to the chemistry of the dust formation process. Spectroscopically, on the other hand, S stars are defined as showing strong bands of $\mathrm{ZrO}$ (cf. Jaschek \& Jaschek 1987). The strong visibility of these bands in a stellar spectrum depends besides the $\mathrm{C} / \mathrm{O}$ abundance ratio also strongly on the $\mathrm{Zr}$ abundance, particular at $T_{\text {eff }} \gtrsim 3000 \mathrm{~K}$ (Piccirillo 1980). Since the Zr abundance can be greatly enhanced by "dredge-up" of freshly synthesized $s$-process elements, also stars with a $\mathrm{C} / \mathrm{O}$ abundance ratio below our lower limit may spectroscopically be classified as $\mathrm{S}$ star. Consequently $\mathrm{C} / \mathrm{O}$ abundance ratios of observed $\mathrm{S}$ stars are from a wider region of $\mathrm{C} / \mathrm{O}$ abundance ratios as that defined above: Smith \& Lambert determined $\mathrm{C} / \mathrm{O}$ abundance ratios down to $\approx 0.5$ for their sample of $\mathrm{S}$ and MS stars. Such spectroscopic $S$ stars with $\epsilon_{\mathrm{C}}<\epsilon_{\mathrm{O}}-2 \epsilon_{\mathrm{Si}}$ should form a normal mineral mixture in their dust shells, in line with the finding that part of the dust shells of $\mathrm{S}$ stars show the same silicate features as dust shells around $\mathrm{M}$ stars, though not very strong ones and that these features disappeare if $\mathrm{C} / \mathrm{O}$ approaches unity (e.g. Chen \& Kwok 1992; Lloyd Evans \& Little-Marenin 1999). Without a detailed modeling of the stellar atmosphere we cannot determine for our synthetic AGB evolution models, which stars spectroscopically would appear as S stars. For this reason we use the chemical definition.

\section{A.2. Dust production by M-stars}

A typical result for the mineral mixture in an oxygen rich wind model is shown in Fig. A.1. The degree of condensation is rather low in a wind with low mass-loss rate, but a variety of dust species can be formed in such winds. At much higher mass-loss rates usually the mineral mixture is dominated by only one species which is the first one which starts to condense in significant amounts (cf. Ferrarotti \& Gail 2002). In the following we do not discriminate observationally between 


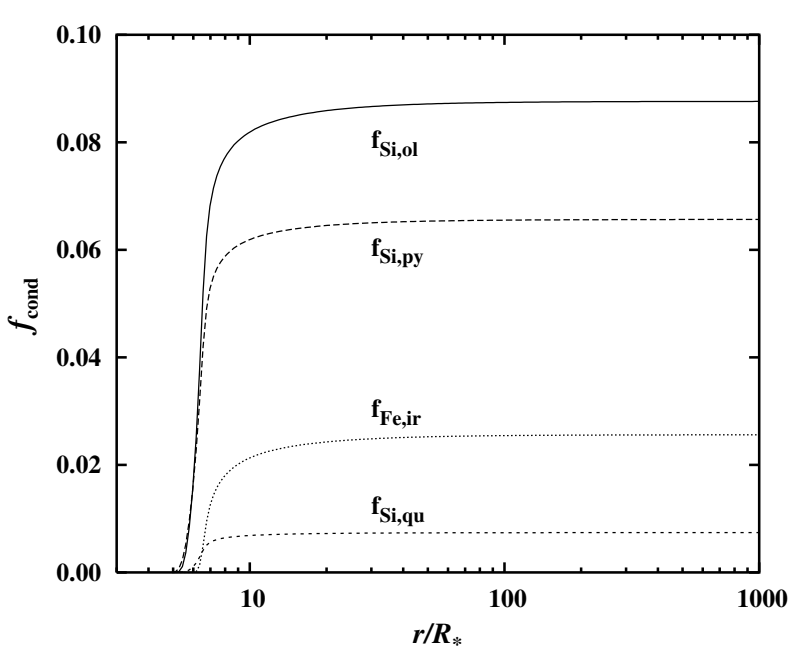

Fig. A.1. Radial variation of the degree of condensation of Si into olivine-, pyroxene-, and quartz-type dust, and of Fe into iron grains. Model with $L_{*}=1 \times 10^{4} L_{\odot}, T_{\text {eff }}=3000 \mathrm{~K}, \dot{M}=3 \times 10^{-6} M_{\odot} \mathrm{yr}^{-1}$ and $\mathrm{C} / \mathrm{O}$ abundance ratio of 0.5 .

the different silicate bearing dust species, since presently it is not possible to discriminate between different silicate species in the ISM. We consider for this reason only the total degree of condensation $f_{\mathrm{Si} \text {,sil }}$, which is defined as the sum of the individual degrees of condensation of Si into olivine-, pyroxene-, and quartz type dust. Similar $f_{\mathrm{Mg} \text {,sil }}$ and $f_{\mathrm{Fe}, \text { sil }}$ are the total degrees of condensation of $\mathrm{Mg}$ or $\mathrm{Fe}$, respectively, into olivineand pyroxene-type dust.

Figures A.2 and A.3 show the variation of the degree of condensation of the main dust forming elements $\mathrm{Si}, \mathrm{Mg}$, and $\mathrm{Fe}$ into dust species with varying mass-loss rates and $\mathrm{C} / \mathrm{O}$ abundance ratios. The general trend is that only some fraction of the condensible elements is really condensed into dust. The degree of condensation is rather low at low mass loss rates and increases to significant depletion of the gas phase only for very high mass-loss rates which are only met in the superwind phase. Such a behavior has been observed in the $\mathrm{SiO}$ lines from circumstellar shells (González et al. 2003).

There is a rapid decline of the amount of silicate dust formed between a $\mathrm{C} / \mathrm{O}$ abundance ratio of about 0.82 and 0.90 , as it is to be expected. Above a $\mathrm{C} / \mathrm{O}$ abundance ratio of 0.90 almost no silicate dust is formed. This results from the decreasing availability of sufficient amounts of oxygen to form the basic building blocks of the silicates, the $\mathrm{SiO}_{4}$-tetrahedron. The results of the calculation show the limit between the standard mineralogy of dust shells around $\mathrm{M}$ stars and the iron dominated dust mixture of $\mathrm{S}$ stars to occur at a $\mathrm{C} / \mathrm{O}$ abundance ratio of $1-2 \epsilon_{\mathrm{C}} / \epsilon_{\mathrm{O}}$. This value is used in our synthetic AGB calculations as the limit between the cases of $\mathrm{M}$ and $\mathrm{S}$ stars.

The high fraction of iron dust formed in the models results from an unrealistically low expansion velocity at the transition to S-star chemistry of the models for the stellar parameters chosen, because extinction by iron is rather inefficient for driving a stellar wind, as can be seen from Fig. 1. Real stars of this elemental composition would have a lower mass or a higher luminosity at this evolutionary stage as that assumed in the present models.

$$
f_{\mathrm{Si}, \mathrm{sil}}
$$

(a)

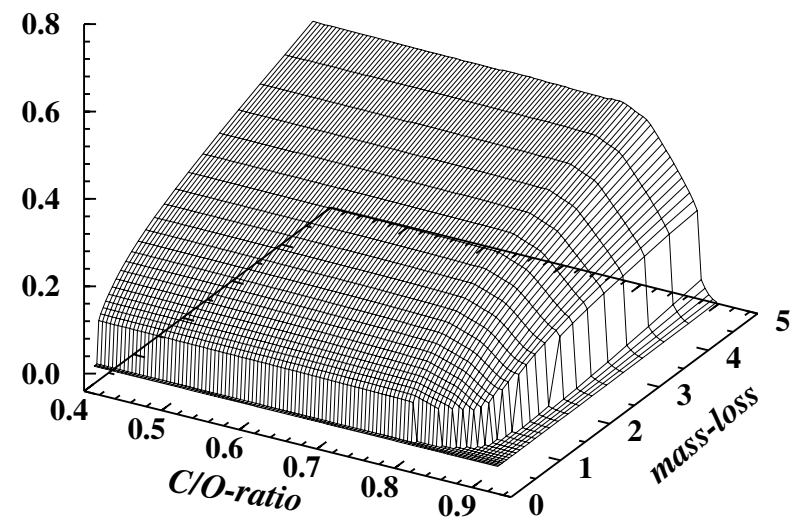

\section{$f_{\mathrm{Mg}, \mathrm{sil}}$}

(b)

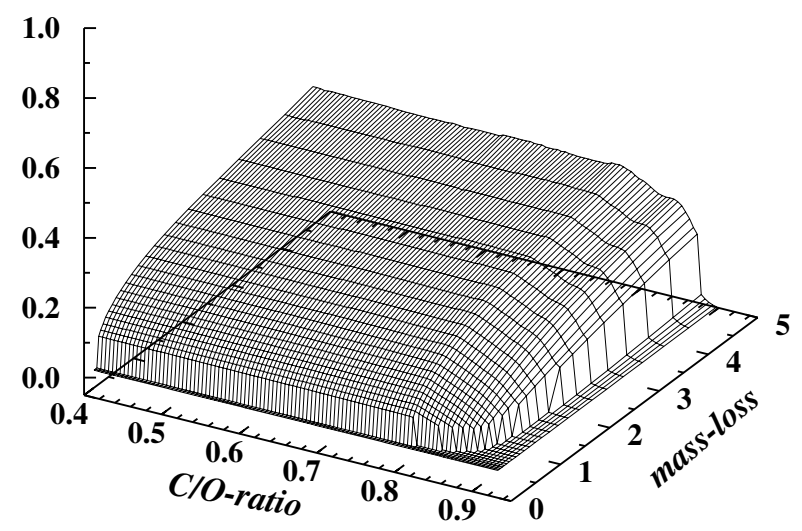

Fig. A.2. Variation of the degree of condensation with $\mathrm{C} / \mathrm{O}$ abundance ratio and mass-loss rate. The mass-loss rate is in units of $10^{-5} M_{\odot} \mathrm{yr}^{-1}$. a) Fraction of $\mathrm{Si}$ condensed into silicate dust species. b) Fraction of Mg condensed into silicate dust species. Models with $L_{*}=1 \times 10^{4} L_{\odot}$ and $T_{\text {eff }}=3000 \mathrm{~K}$.

\section{A.3. Dust production by C-stars}

Figure A.4 shows the variation in the degree of condensation of the main dust-forming elements $\mathrm{C}, \mathrm{Si}$, and $\mathrm{Fe}$ into dust species with varying mass-loss rates and $\mathrm{C} / \mathrm{O}$ abundance ratios. The degree of condensation of carbon dust refers to the total carbon abundance, not to the excess carbon that not bound in $\mathrm{CO}$.

With our model's assumptions on carbon growth, the SiC dust is the first dust component to start growing. Since the extinction by $\mathrm{SiC}$ alone is not very efficient in driving a stellar outflow (cf. Fig. 1), the condensation of SiC does not suffice for driving the dusty gas to highly supersonic outflow velocities. $\mathrm{SiC}$ therefore grows in a region of moderate outflow velocity, which results in a rather high degree of condensation.

If the carbon starts to condense at a somewhat lower temperature, its high extinction drives the outflow to the observed high outflow velocities. This prevents the formation of a significant amount of iron dust, since iron growth becomes possible only at a significantly lower temperature than the one where carbon condensation commences. Then the gas is already too diluted for efficient iron dust formation. Only at $\mathrm{C} / \mathrm{O}$ abundance 


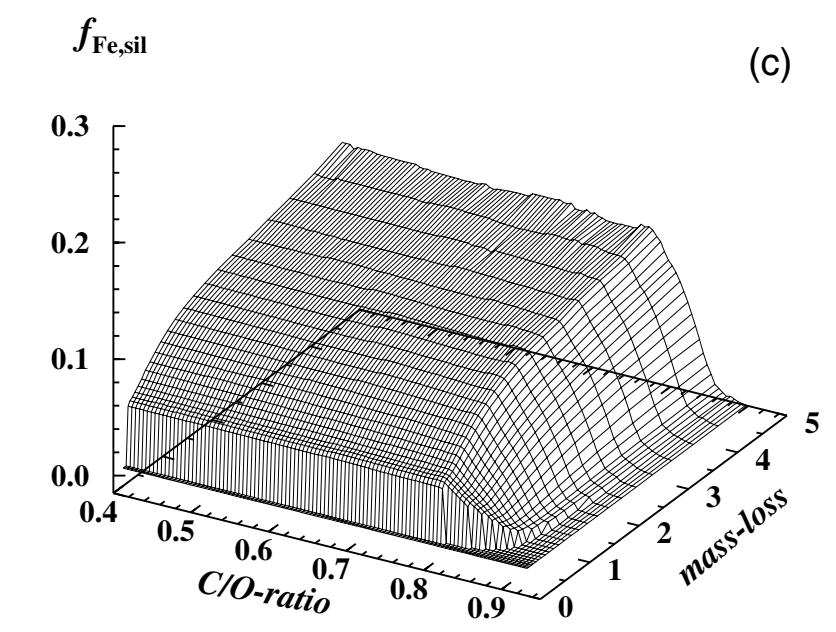

$$
f_{\mathrm{C}}
$$

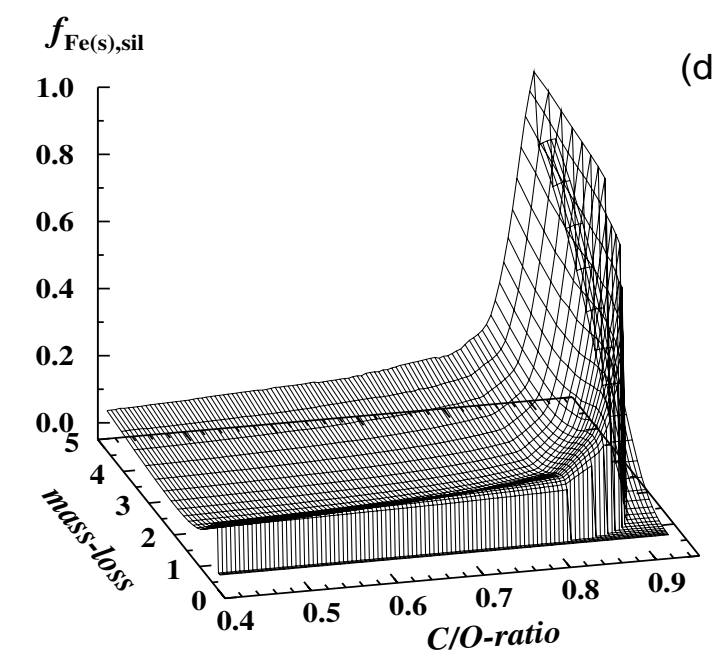

Fig. A.3. As in Fig. A.2 c) Fraction of Fe condensed into silicate dust species. d) Fraction of Fe condensed into metallic iron.

ratios close to unity, where carbon dust is formed only in small quantities, is iron an abundant dust species.

\section{Appendix B: The initial-to-final mass relation}

The core mass at the end of the AGB evolution is essentially identical to the masses of the final White Dwarf remnant of the star. The small increase in core mass during the subsequent evolution through the PN stage to the White Dwarf cooling sequence can be neglected. The relation between the core mass at the end of AGB evolution to the initial main sequence mass of the star can be compared to the empirically determined initial-to-final mass relation for White Dwarfs in stellar clusters. This is a test of the reliability of the models we calculated.

This relation has been discussed several times over the years by Weidemann, most recently in Weidemann (2000), which also gives references for the empirical data material. Some improvements in observed and derived final and initial masses are given in Claver et al. (2001). In Fig. B.1a, we plot the empirical initial-to-final mass relation (full line) and the data for White Dwarfs taken from the sources given in Weidemann (2000) and in Claver et al. (2001). For clarity we do not show error bars for the initial and final masses
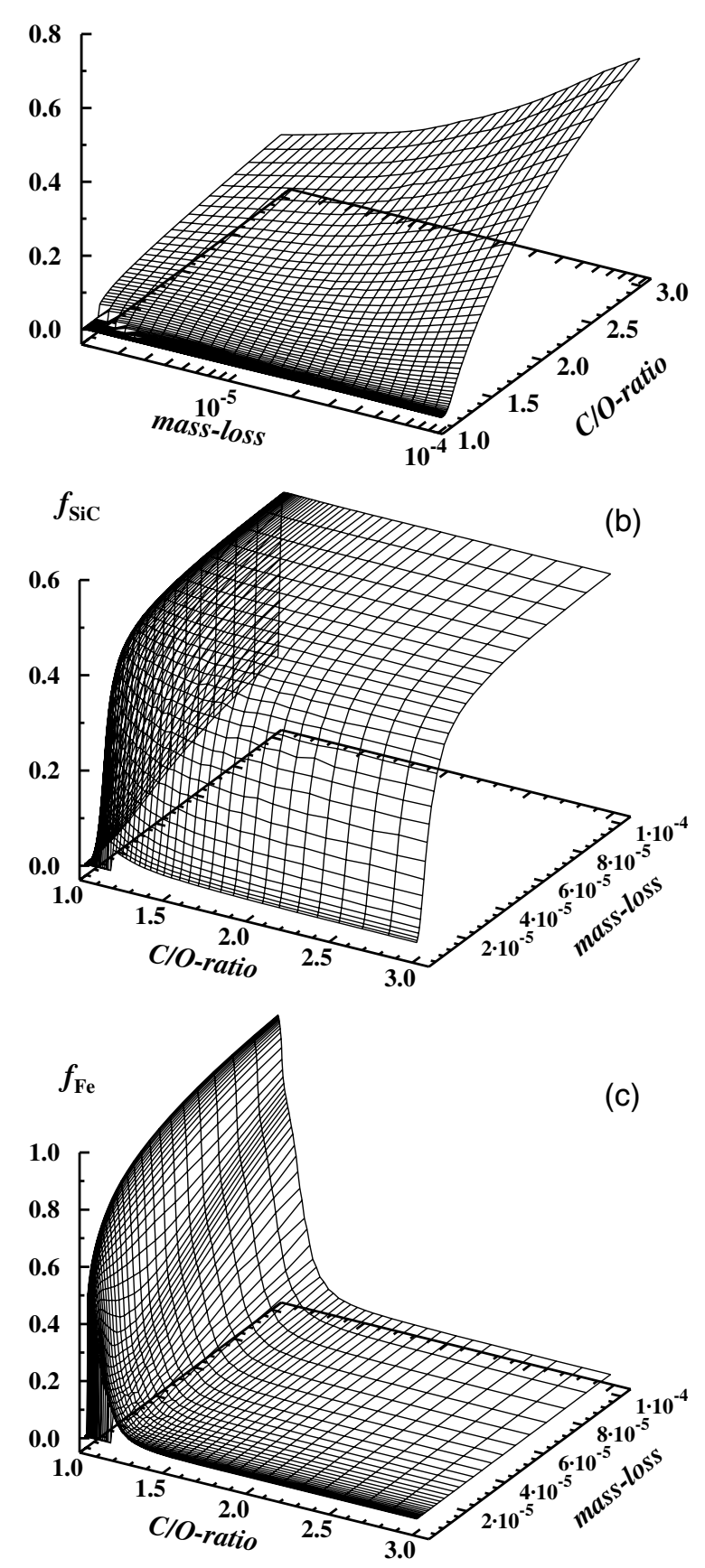

Fig. A.4. Variation of the total degree of condensation with $\mathrm{C} / \mathrm{O}$ abundance ratio and the mass-loss rate. a) Degree of condensation of $\mathrm{C}$ into solid carbon. b) Degree of condensation of $\mathrm{Si}$ into $\mathrm{SiC}$. c) Degree of condensation of Fe into solid iron. Models with $L_{*}=1 \times 10^{4} L_{\odot}$ and $T_{\text {eff }}=2500 \mathrm{~K}$.

of the White Dwarfs, which in most cases are considerable. The dashed lines are the initial-to-final mass relations for models with $Z=0.008,0.02,0.04$, as obtained from our model calculations with the Karakas et al. (2002) "dredge-up" prescription. The dotted lines are the core masses at the onset of thermal pulsing determined from the models of Schaller et al. (1992), Schaerer et al. (1993), and Charbonnel et al. (1993), 
A. S. Ferrarotti and H.-P. Gail: Dust production by AGB stars, Online Material p 5

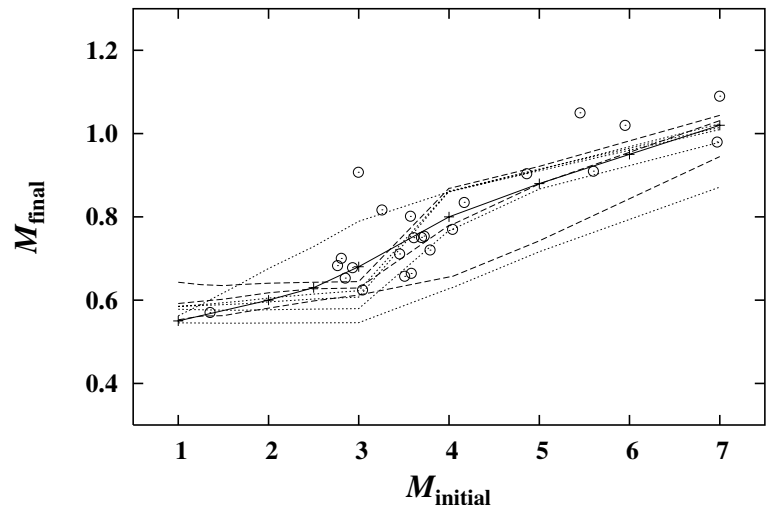

Fig. B.1. Relation between the initial stellar mass and the final mass of the White Dwarf remnant. Models are calculated with the Karakas et al. (2002) parametrization of the "dredge-up" efficiency $\lambda$. Dashed lines: the initial-to-final mass relation according to the model calculation for three different metallicities $(Z=0.008,0.02,0.04$, from top to bottom). Dotted lines: mass of the $\mathrm{C}+\mathrm{O}-$ core at the onset of thermal pulsing for different metallicities. Full line: the empirical initial-to-final mass relation determined from White Dwarfs in stellar clusters (Weidemann 2000). Circles: the individual stars on which the empirical relation is based on (cf. Weidemann 2000).

which we take as initial models for our synthetic AGB evolutionary models.
For stars that are more massive than $\approx 2.5 M_{\odot}$, the final core mass at the end of AGB evolution is not much higher than at the onset of TP-AGB evolution, because of the high dredge-up efficiency $\lambda$ for intermediate mass stars according to the parametrization of Karakas et al. (2002), which mixes most of the He that is freshly produced during the inter-pulse phase to the envelope. In effect, the H-burning in the interpulse phase adds little He to the core after the onset of thermal pulsing. This high efficiency of "dredge-up" for intermediate mass stars is also found in other calculations (see the discussion in Weidemann 2000). For the intermediate mass stars, our results for $Z=0.02$ closely follow the empirical initial-to-final mass relation which is determined for solar metallicity stars. This seems to indicate that our synthetic AGB models, with the Karakas et al. (2002) parametrization for $\lambda$, reproduce the real masses of the White Dwarf remnants for intermediate mass stars.

For low mass star models calculated with the Karakas et al. (2002) values for $\lambda$, the final core mass seems to be higher than the single empirically-determined data point, but this does not suffice for constraining the models in this mass-region. Our results for low mass stars are, in any case, within the limits of the empirical White Dwarf mass distribution (cf. Weidemann 1990; Finley et al. 1997). 